\title{
Physiological Properties of Vestibular Primary Afferents that Mediate Motor Learning and Normal Performance of the Vestibulo-ocular Reflex in Monkeys
}

\author{
Helen M. Brontë-Stewart and Stephen G. Lisberger \\ Department of Physiology, W. M. Keck Foundation Center for Integrative Neuroscience, and Neuroscience Graduate \\ Program, University of California, San Francisco, California 94143
}

We have used electrical stimulation of the vestibular apparatus to reveal parallels between the physiological responses of the vestibular afferents activated at different currents and the properties of the evoked eye movements before and after magnifying spectacles had been used to cause motor learning in the vestibulo-ocular reflex (VOR). Stimulation with the lowest currents caused little or no eye motion, but activated all the afferents with irregular spontaneous discharge, low sensitivities to head velocity, and highly phasic responses during rapid head turns. Stimulation with moderate currents caused substantial eye motion that was weakly affected by motor learning; these currents activated afferents with a wide range of physiological properties, including many that had intermediate discharge regularity, high sensitivity to head velocity, and clear phasic responses during rapid head turns. Stimulation at still higher currents caused still larger eye movements that were strongly altered by motor learning; these currents activated primarily afferents that had regular spontaneous discharge, lower sensitivities to head velocity, and tonic responses during rapid head turns. Stimulation at the highest currents did not cause any further increment in the amplitude of the evoked eye movement, but activated the afferents with the most regular spontaneous discharge and the lowest sensitivities to head velocity. The data imply that the VOR pathways receive substantial vestibular inputs from afferents with a middle range of thresholds for electrical stimulation. These afferents have a wide range of physiological properties, including a large group that shows substantial phasic responses during rapid head turns. The data also suggest that only a subset of these afferents, primarily those with more regular spontaneous discharge, project into the VOR pathways that are modified in association with motor learning.

[Key words: vestibular nerve, vestibulo-ocular reflex, motor learning, plasticity, oculomotor system, alert rhesus monkeyl

\footnotetext{
Received Mar. 26, 1993; revised Aug. 11, 1993; accepted Aug. 13, 1993.

We are grateful to Drs. Sascha du Lac and Dianne Broussard for helpful comments on earlier versions of the manuscript, and to Dr. Richard Krauzlis for insightful comments during the experiments. We also thank Terri Pavelko and Josh Schwartz for technical assistance. This rescarch was supported by NIH Grants K11-EY00302 and R37-EY03878, and by a Development Award from the McKnight Neuroscience Endowment Fund.

Correspondence should be addressed to Stephen G. Lisberger, Department of Physiology, Box 0444, UCSF, San Francisco, CA 94143-0444.

Copyright (C) 1994 Society for Neuroscience $0270-6474 / 94 / 141290-19 \$ 05.00 / 0$
}

Each modality of sensory inputs to the brain is subserved by primary afferents that carry a wide range of signals. In the primate visual system, for example, the magnocellular and parvocellular pathways provide quite different kinds of information about the visual scene and much has been learned about the functions of these two parallel sensory channels (Merigan and Maunsell, 1993). The somatic and proprioceptive sensory systems also show wide diversity in the kind of information that is transmitted by afferents from each modality. For example, phasic information about the velocity of muscle stretch is transmitted by the Ia afferents from muscle stretch receptors while more tonic information about muscle length is encoded in the steady and regular firing of the group II afferents (Matthews and Stein, 1969a,b). However, little is known about how these different proprioceptive afferent signals are channeled to different parts of the sensory and motor systems. Our goal has been to use the vestibular inputs for stabilizing gaze as a model system to investigate the general question of how the sensory inputs from an individual modality are channeled into parallel central pathways that subserve different functions.

Vestibular afferents provide the inputs for a number of different vestibulomotor reflexes. When a dancer is learning to execute a series of pirouettes, for example, each movement depends on the parallel operation of the three vestibular reflexes. The vestibulospinal reflex (VSR) maintains the orientation of the body in space, the vestibulocollic reflex (VCR) compensates for body movements and stabilizes the orientation of the head in space, and the vestibulo-ocular reflex (VOR) compensates for head movements and stabilizes eye movements with respect to the world. These different reflexes must overcome quite different physical forces and therefore require control signals with different dynamics. For example, the VOR must overcome the viscoelastic properties of the orbit (Robinson, 1970; Skavenski and Robinson, 1973) while the VCR must generate forces to overcome the inertial properties of the head and neck (Peterson et al., 1981).

The different control signals needed for each vestibular reflex could be provided by the wide range of physiological responses of the different afferents from an individual vestibular receptor. Vestibular afferents from each individual semicircular canal differ in the regularity of spontaneous firing rate, in the rate and degree of adaptation to constant angular head acceleration, and in the sensitivity and phase shift during sinusoidal head rotation (Fernandez and Goldberg, 1971; Goldberg and Fernandez, 1971 a,b; Estes et al., 1975; Tomko et al., 1981). Afferents also vary in the trajectory of their firing rate during rapid head turns; 
some exhibit a pronounced phasic component in the response while others show purely tonic responses (Lisberger and Pavelko, 1986). Some of the physiological properties are correlated. For example, afferents with more irregular spontaneous firing tend to show greater adaptation to constant angular head acceleration (Goldberg and Fernandez, 1971 a; Blanks et al., 1975; Tomko et al., 1981), larger phase leads with respect to head velocity at frequencies of head rotation above $1 \mathrm{~Hz}$ (Fernandez and Goldberg, 1971), and more phasic responses to rapid head turns (Lisberger and Pavelko, 1986).

A number of previous approaches have provided some indirect evidence that vestibular inputs with different dynamics are channeled toward specific vestibular reflexes according to their dynamic response properties. Comparisons of the responses to head rotations of primary afferents (Goldberg and Fernandez, 1971a) and of neurons that receive monosynaptic inputs from the primary afferents (Precht and Shimazu, 1965; Shimazu and Precht, 1965; Melvill Jones and Milsum, 1970; Shinoda and Yoshida, 1974) have demonstrated similar bimodal distributions of the time constants of their responses to steps of head acceleration. Comparisons of the responses of the same groups of primary and secondary neurons to electrical stimulation of the vestibular apparatus (Goldberg et al., 1987; Highstein et al., 1987) have established correlations between the thresholds of central vestibular neurons and of vestibular afferents with different physiological propertics. These authors found that interneurons in the disynaptic VOR pathways to the oculomotor nuclei received less input from afferents with irregular spontaneous firing than did interneurons in pathways to the spinal cord. Finally, measures of reflex output have provided some information about the afferents that project into the pathways that subserve the VOR and VCR. Minor and Goldberg (1991) demonstrated that reversible ablation of the afferents with irregular spontaneous discharge did not affect the VOR evoked by head turns in the dark, suggesting that these afferents do not project into VOR pathways. Bilotto et al. (1982) compared the dynamics of the VCR to the responses of vestibular afferents and central vestibular neurons (Ezure and Sasaki, 1978; Wilson et al., 1979) and concluded that the VCR pathways receive inputs predominantly from the group of afferents that has more phase lead during sinusoidal vestibular stimulation.

Studies of motor learning in the VOR have suggested that different afferents could serve different functions in a single reflex. In normal monkeys, rotatory head turns in one direction evoke compensatory eye movements in the other direction. The gain of the VOR, defined as eye speed divided by head speed during passive head rotation in darkness, is close to 1.0 . If a monkey wears magnifying or miniaturizing spectacles for several days, then the VOR undergoes motor learning such that the gain of the VOR increases or decreases (Miles and Eighmy, 1980 ) to values as high as 1.8 or as low as 0.25 (Lisberger and Pavelko, 1986). Comparison of the eye movements evoked by rapid head turns when the gain of the VOR is low, normal, and high has revealed at least two components of the VOR. The two components differ in the degree to which they are modified in association with changes in the gain of the VOR, in the latency of their earliest responses (Lisberger, 1984), and in the dynamics of the signals that drive them (Lisberger and Pavelko, 1986). These authors accounted for the properties of the different components of the VOR with a hypothesis in which afferents with more phasic response properties would provide inputs to VOR pathways that are not modified in association with motor learn- ing while afferents with more tonic response properties would provide inputs to modified VOR pathways. In a modeling report, Lisberger and Sejnowski (1992) suggested that both groups of afferents contribute to motor learning and showed how learning could be accomplished by changing the balance of tonic and phasic vestibular inputs to the cerebellum. Thus. different classes of afferents could play different roles in motor learning, even though motor learning in the VOR clearly occurs in the CNS and is not expressed in the firing rate of primary afferents themselves (Miles and Braitman, 1980).

Although the experiments outlined above have suggested that different vestibular afferents subserve different functions, the evidence is largely indirect. Our goal was to generate more direct evidence concerning the properties of the primary afferents that drive different components of the VOR. Our approach was to use electrical stimulation of the vestibular apparatus to make a direct comparison between the recruitment pattern of afferents and the eye movements evoked by the same electrical stimulus. By analyzing the eye movements evoked at different currents and comparing their properties to the physiological responses of the afferents activated at the same currents, we have been able to deduce the physiological response properties of afferents that are responsible for the VOR. In an earlier publication (Broussard et al., 1992), we had demonstrated that motor learning in the VOR has a small but reliable effect on the eye movements evoked by clectrical stimulation of the vestibular apparatus with single pulses. However, this earlier report did not attempt to deduce the relative contributions of different afferents to the learned component of the response. In the present article, we analyze the larger effect of motor learning in the VOR on the eye movements evoked by trains of stimuli at different currents and we use that analysis to draw conclusions about the physiological response properties of afferents that contribute to the learned response.

\section{Materials and Methods}

Monkey training and general experimental procedure. Experiments were conducted on four male rhesus monkeys that weighed between 6 and 9 $\mathrm{kg}$. For behavioral training and daily experiments, the monkeys moved voluntarily from their home cages to specially designed primate chairs. Initially, each monkey was trained to perform a reaction time task (Wurtz, 1969). After the monkey had learned this task, he was anesthetized with halothane and sterile surgical procedure was used to implant a scleral search coil on one eye (Judge et al., 1980) so that we could use the magnetic search coil method to monitor eye position. At the same time three or four bolts were implanted in the skull to secure a dental acrylic pedestal that provided a receptacle for painlessly restraining the monkey's head during experiments and for mounting goggles that magnified or miniaturized vision (Lisberger and Pavelko, 1986).

After the monkey had recovered from surgery, further training was conducted. The implanted receptacle was used to secure the monkey's head to the ceiling of the primate chair, the chair was bolted to a motorized turntable, and a cube containing two pairs of 18 inch coils was lowered over and secured to the chair. The coils provided the magnetic field needed to measure horizontal and vertical eye position. Natural vestibular stimulation was provided by a servo-controlled turntable (Contraves-Goertz model $813,20 \mathrm{ft} / \mathrm{lb}$ peak torque) that oscillated the chair, the monkey, and the field coils together about a vertical axis. The monkey's head was held in the stereotaxic plane such that horizontal turntable rotation activated the horizontal semicircular canals almost maximally but also activated the vertical semicircular canals weakly The monkey was trained to fixate and track a target that was created by projecting a $0.5^{\circ}$ white circular spot onto the back of a tangent screen, which was $114 \mathrm{~cm}$ from him. The eye coil was calibrated initially by having the monkey perform the bar-press task while the target was at different, known positions. The bar was then removed and the monkey 
was rewarded at intervals of $1.5 \mathrm{sec}$ for kecping his cyes within $2-3^{\circ}$ of the target.

Preparation for stimulation of the vestibular apparatus. When the monkey was proficient at fixating and tracking the target, we performed two additional surgeries to prepare for single-unit recording from the vestibular nerve and for electrical stimulation of the vestibular apparatus. Halothane anesthesia and sterile surgical technique were used for both procedures. In one procedure, we used stereotaxic techniques to implant a stainless steel cylinder on the skull for introducing microelectrodes into the region of the vestibular nerve (Lisberger and Pavelko, 1986). In the other procedure, we used a postauricular approach that we have described previously (Broussard et al., 1992) to place a stimulating electrode in the perilymphatic compartment of the superior semicircular canal. Several observations suggest that our electrode implants did not disrupt the physiological function of the horizontal canal. First, measurement of eye movement an hour after completion of surgery revealed that the gain of the VOR was close to 1.0 in darkness and that the monkeys showed neither nystagmus nor head tilt. Second, all of our monkeys achieved a VOR gain greater than 1.7 after adaptation to magnifying spectacles; we have found this to be a reliable indication that both horizontal semicircular canals are functioning normally (Broussard et al., 1992). Third, horizontal canal afferents were activated by electrical stimulation of the superior semicircular canal with currents as low as $20 \mu \mathrm{A}$ and their responses to natural head turns were normal. We assume that the electrodes activate horizontal canal afferents at low currents because of the proximity of the ampullated ends of the horizontal and superior semicircular canals. Thus, implants in the superior canal provide a way to activate afferents from the horizontal canal at low currents without compromising the mcchanical function of the horizontal canal.

Recordings from primary afferent fibers. We used glass-insulated platinum-iridium microelectrodes to make extracellular recordings from axons within the vestibular nerve. These microelectrodes were lowered into the brain through the chronically implanted cylinder and were driven with a hydraulic microdrive through the cerebellar ventral paraflocculus and flocculus to the vestibular nerve (Lisberger and Pavelko, 1986). Proximity to the nerve was marked by silence as the electrode left the overlying cerebellum. Axonal recordings were recognized by triphasic action potentials with an initially positive deflection (about $90 \%$ of fibers) or by brief negative potentials (about $10 \%$ of fibers).

We found that electrodes with fine, sharp tips about $7 \mu \mathrm{m}$ in length were superior for isolating single afferents, especially those with the highest thresholds for activation by clcctrical stimuli. We also found that significant forward and backward motion of the electrode within the nerve fascicles was often necessary to isolate a single fiber. Unit spikes were triggered with a standard amplitude-window discriminator. The time of each spike was recorded to the nearest $10 \mu \mathrm{sec}$ by Schmitt trigger inputs to the computer. Isolation of the action potentials from a single fiber was confirmed by inspection of the waveforms of the spikes on a digital storage oscilloscope that was triggered by the acceptance pulse from the discriminator.

Measurement and long-term modification of the gain of VOR. The VOR was subjected to motor learning by fitting each monkey with magnifying or miniaturizing spectacles that were worn while the monkey moved freely in his home cage (Lisberger and Pavelko, 1986). The spectacles were customized for each monkey so that they could be worn comfortably for wccks at a timc. The monkey's head was secured at least once a day so that the spectacles could be removed and cleaned and the face could be inspected for any sign of pressure from the spectacle frames. The performance of the VOR was then measured by recording and analyzing the eye movements evoked in the dark by a sequence of brief pulses of head velocity. Pulses of head velocity were driven by the digital-to-analog converters of a computer. Each pulse consisted of rapid acceleration at $600 \% \mathrm{sec}^{2}$ for $50 \mathrm{msec}$, rotation at $30 \% \mathrm{sec}$ for $200 \mathrm{msec}$, and rapid deceleration back to $0 \% \mathrm{sec}$. Alternation of leftward and rightward pulses at intervals of $1096 \mathrm{msec}$ resulted in a trapezoid of angular head position. In the intervals between the pulses of head velocity, the background was dark and the monkey was required to fixate on a stationary target. The target was turned off $100 \mathrm{msec}$ before the turntable began to move and came back on $100 \mathrm{msec}$ after the head velocity had rcturncd to zcro.

Experimental protocol: effect of changes in the gain of the VOR on eye movements evoked by electrical stimuli. In this experiment, which was conducted on all four monkeys, we modified the gain of the VOR and recorded the effects on the eye movements evoked by electrical stimulation of the labyrinth over a time course of several weeks. In each daily session, we first fixed the monkey's head to the ceiling of the chair, removed the spectacles, and measured the gain of the VOR for pulses of head velocity. Next, we recorded the eye movements evoked by single electrical pulses applied to the vestibular apparatus. Each pulse provided a balanced biphasic current for a total duration of $400 \mu \mathrm{sec}$. The stimuli were provided by a voltage-controlled constant current stimulus isolation unit (BAK model BSI-1). Our earlier experiments showed that stimulation of the vestibular apparatus evokes movements that follow the same trajectories in both eyes, although the absolute amplitude is often larger in the eye ipsilateral to the stimulating electrode (Broussard et al., 1997). In the present study, eye movements were recorded from the eye contralateral to the ear being stimulated to avoid recording artifacts caused by the activation of facial muscles due to current spread to the facial nerve. Stimuli were applied in sets of 25 pulses of the same current and with an interpulse interval of $200 \mathrm{msec}$. Each set provided pulses at a different current starting from below the threshold for evoking an eye movement and increasing in $50 \mu \mathrm{A}$ increments to values that were clearly high enough to saturate the peak eye velocity. During electrical stimulation, the monkey was rewarded for fixating a stationary target. Because the pulses were widely spaced and each pulse produced only a tiny displacement in eye position, the application of electrical stimuli did not affect the monkey's ability to fixate the target and receive rewards. Finally, we applied a series of 12-15 trains of pulses at each of the same currents. The trains consisted of 16 pulses at intervals of 5 msec and the interval between trains was approximately $1 \mathrm{sec}$. The trains of pulses tended to drive the monkey's fixation off target by up to $5^{\circ}$, but the presence of a visual stimulus did not affect the response because the duration of the train was $75 \mathrm{msec}$ and the response was therefore over before there had been time for visual feedback.

Baseline data at a normal VOR gain were collected for at least 3-7 d before each monkey was fitted with magnifying or miniaturizing spectacles. After the spectacles were initially fitted, the monkey was returned to his home cage and allowed to move around naturally. Data on the responses to natural and electrical stimulation of the vestibular apparatus were collected $2-3 \mathrm{hr}$ after the spectacles were first placed on the monkey and approximately daily thereafter until the gain of the VOR had clearly settled at an asymptote. The asymptotic value of the gain of the VOR ranged from 1.76 to 1.82 for magnifying spectacles and from 0.27 to 0.32 for miniaturizing spectacles. Experiments were run for 3-7 $\mathrm{d}$ at each asymptote before the spectacles were removed, the gain of the VOR was allowed to return to normal for at least $3 \mathrm{~d}$, and the opposite set of lenses was placed on the monkey.

Experimental protocol: recordings from primary afferent fibers. In this experiment, which was conducted on two of the monkeys, we recorded the responses of vestibular primary afferents to natural and electrical stimulation of the vestibular apparatus when the gain of the VOR was normal. Before each daily experiment, we measured the eye movements evoked by stimulation of the labyrinth with single electrical pulses over a range of currents. Analysis of these data allowed us to identify any changes in the efficacy of our stimulating electrodes and to normalize the stimulation current for comparison of data obtained on different days and in different monkeys. We then introduced microelectrodes into the vestibular nerve and searched during head rotation in the horizontal plane for afferents that showed increased firing during ipsiversive head rotation and therefore innervated the horizontal semicircular canal. Afferents from the posterior semicircular canal would have shown weak modulation of firing rate during horizontal head rotation, with increased firing during contraversive motion. Afferents from the superior canal should have been unresponsive to horizontal head motion because the electrode mechanically plugged the canal. Afferents from the otolith organs should have been unresponsive because horizontal angular head rotation does not provide an adequate stimulus for the otoliths.

After the spikes from a horizontal canal afferent were isolated, we stimulated electrically over a range of currents to determine the threshold for activation of the afferent. In early experiments, we found a steep relationship between the probability of activation of an individual afferent and the stimulation current. Consequently, we developed a protocol that allowed us to find the relevant current range quickly and then to vary current in small steps within that range. After we found the relevant range of currents, we recorded data for quantitative analysis, starting at a current that never activated the fiber and increasing the current in increments of $10 \mu \mathrm{A}$ until every stimulus evoked a spike. At each current we applied a series of single pulses with an interpulse 
interval of $200 \mathrm{msec}$. In our early experiments, we used 200 pulses at each current, but we reduced that number in later experiments after we had ascertained that reliable estimates of threshold could be obtained with just 100 pulses at each current.

Previous experiments have shown that the current required to activate a primary vestibular afferent depends on the interval between the last action potential and the stimulus (Goldberg et al., 1984). We therefore synchronized each stimulus pulse to a naturally occurring action potential so that it occurred at a delay that corresponded to $40-50 \%$ of the afferent's interspike interval (ISI). To ensure that we maintained an interval of approximately $200 \mathrm{msec}$ between pulses, one stimulus was enabled every $200 \mathrm{msec}$ but was not triggered until the selected delay after the next spontaneous action potential. For 40 fibers, we estimated how the threshold varied as a function of the delay between the previous spike and the application of the stimulus. We varied the delay from about $20 \%$ to about $70 \%$ of the ISI. For each delay, we estimated the threshold current by adjusting the current as we viewed the evoked spikes on an oscilloscope. Once we had found the current that activated the fiber approximately $50 \%$ of the time, we recorded the afferent's response to 30 pulses so that data analysis could verify our estimates.

Once we had determined an afferent's threshold for electrical stimulation, we recorded its physiological response properties. First, we recorded its response to 10 cycles of horizontal sinusoidal head oscillation at $0.5 \mathrm{~Hz}$ and to 50 cycles of sinusoidal oscillation at $4 \mathrm{~Hz}$. This provided about $20 \mathrm{sec}$ of data at each frequency. Both stimuli provided a peak-to-peak head velocity of $60 \% \mathrm{sec}$. Then, we then recorded $10-20$ $\mathrm{sec}$ of the afferent's spontaneous activity with the head stationary. Finally, we recorded 2 min of the spike train evoked by a set of the pulses of head velocity that were described earlier.

Data acquisition. Experiments were run under the control of a DEC $11 / 23$ computer that digitized voltages proportional to horizontal eye position, target position, and head velocity at a rate of $500 \mathrm{samples} / \mathrm{sec}$ per channel. The computer recorded the times of occurrence of pulses from the window discriminator to the nearest $10 \mu \mathrm{sec}$ and recorded the time of application of electrical stimuli to the labyrinth to the nearest $100 \mu \mathrm{sec}$. A tachometer on the turntable provided a direct measure of angular head velocity, and a precision potentiometer attached to the shaft of the turntable measured angular head position. Signals related to eye velocity were obtained by using two analog differentiators that had different filtering properties. One differentiator passed frequencies up to $50 \mathrm{~Hz}$ and yielded signals with a high signal-to-noise ratio but with a highly filtered profile. The other differentiator passed frequencies up to $100 \mathrm{~Hz}$ and therefore had a lower signal-to-noise ratio but provided a more accurate index of the response latency and of the peak eye velocity for the short-duration responses evoked by stimulation of the labyrinth with single electrical pulses.

Electrical stimulation of the vestibular apparatus caused a large field potential in the vestibular nerve that was a potential source of artifacts in the recorded spike train. Evoked action potentials fell within or just after the field potential, so that it was not possible to tell from the train of acceptance pulses whether the field potential or the spike had triggered the discriminator (see example in Fig. $1 B$ ). To provide data that would allow us to solve this problem, we also sampled the raw spike train at $50 \mathrm{kHz}$ for $40 \mathrm{msec}$ surrounding each stimulus.

Data analysis. The eye movements evoked during the VOR were analyzed by aligning the responses to identical pulses of head velocity and averaging head velocity and eye velocity. Responses were included in the averages only if the monkey had not made any saccadic eye movements between the onset of head motion and the beginning of the deceleration back to zero head velocity. The gain of the VOR was then calculated from the averaged records as the mean eye speed divided by the head speed in the interval $100-200 \mathrm{msec}$ after the onset of the vestibular stimulus.

To analyze the eye movements evoked by single electrical pulses, we averaged the responses to 25 pulses at each current. First, we displayed the data on the video screen of the computer so that responses could be excluded from analysis if they were contaminated by small saccades or if the monkey was not looking at the target. We then aligned the traces at the onset of each stimulus and calculated the mean and standard deviation of eye position and eye velocity in $1 \mathrm{msec}$ intervals, from 20 msec before to $80 \mathrm{msec}$ after the onset of the stimulus. We used a similar procedure to analyze the eye movements evoked by trains of pulses, except that we averaged the responses to only 10 trains. We determined the time of onset of each eye movement response by displaying the average of eye velocity on a video screen and using keystrokes to run
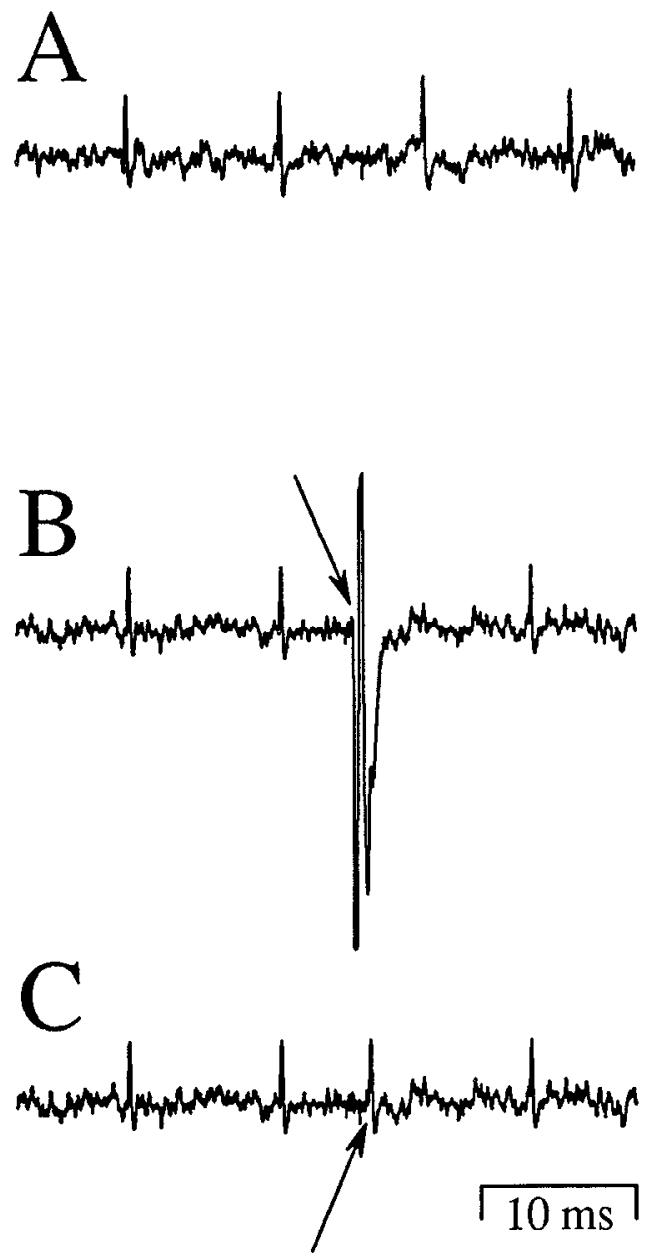

Figure 1. Methods used to subtract the stimulus artifact and field potential from the unit recordings during electrical stimulation of the vestibular apparatus with single pulses. $A$, Extracellular recording from a vestibular axon when no stimulus was applied showing the natural firing pattern of an afferent with regular spontaneous discharge. $B$, Extracellular recording from the same afferent showing an instance when it was activated by stimulation with a single pulse, but the evoked spike was almost entirely obscured by the evoked field potential. $C$, The same trace as in $B$ after the stimulus artifact had been subtracted. The latency of activation was measured as the time between the downward arrow in $B$ and the upward arrow in $C$. Upward deflections of the records show positive potentials.

a cursor along the trace. The latency was measured as the interval between the application of the stimulus pulse and the time of the last sample before eye velocity began a continuous increase toward peak eye velocity.

Before analyzing the responses of primary afferents to single electrical pulses, we formed a template of the evoked potential by averaging 25 traces of the raw spike train in which there was clearly no evoked spike. For afferents with regular or intermediate discharge regularity, the spontaneous spikes were almost equally spaced (Fig. $1 A$ ) and we were able to recognize such traces by the time of the occurrence of the next spike after the stimulus. If a stimulus did not evoke a spike, then the spike after the stimulus artifact occurred at the expected time and the spontaneous spike train was not disturbed. If a stimulus evoked a spike, then the discharge of the afferent was reset so that the next spike was displaced by one spontaneous interspike interval from the evoked spike (Fig. $1 B, C)$. For afferents with irregular spontaneous firing rates, the stimulation current and therefore the amplitude of the field potential was much smaller, so we could see the evoked spikes superimposed on the field potential.

We then aligned the template with each high-speed sample and subtracted the template from the high-speed sample. Figure $1 B$ shows an 
example where an evoked spike was buried in the stimulus artifact and field potential but was revealed (Fig. $1 C$ ) by subtracting the template. We used a cursor to guide the computer in correcting the train of acceptance pulses from the hardware discriminator. This included the deletion of any extra events caused by the stimulus artifact and field potential and the addition of any evoked spikes that had been missed by the hardware discriminator because they were too close to the field potential. Finally, we aligned the responses to all the pulses at a given current on the time of stimulation and made histograms by accumulating in $0.1 \mathrm{msec}$ bins the spikes recorded from each afferent during stimulation at each current.

For each afferent, we measured the latency of the response to electrical stimulation of the vestibular apparatus directly from the high-speed samples of the unit data. The high-speed records of the raw unit data were displayed on a video screen and a cursor was used to measure the time from the start of the stimulus artifact (arrow, Fig. $1 B$ ) to the start of the evoked action potential (arrow, Fig. $1 C$ ). Ten to fifteen measurements were averaged for each fiber. The latencies were measured at the stimulation current that activated the fiber $50 \%$ of the time.

We quantified the regularity of each afferent's spontaneous discharge by calculating the mean firing rate of the afferent and the coefficient of variation $(\mathrm{CV})$, defined as the standard deviation divided by the mean of the interspike interval (ISI). We then used equations from Goldberg et al. (1984) to normalize the $\mathrm{CV}$ for the resting rate and obtain a measure called CV*. Although the equations for normalizing the coefficient of variation were derived in experiments on squirrel monkeys, we think their use in rhesus monkeys is justified by the similarity of the physiological responses of vestibular afferents in these two species.

We followed standard procedures (Lisberger and Miles, 1980; Lisberger and Pavelko, 1986) to analyze the spike trains evoked by sinusoidal head rotation. We divided each cycle of the data into 512 bins and averaged the firing rate and head velocity for 10 cycles of oscillation at $0.5 \mathrm{~Hz}$ and for 50 cycles of oscillation at $4 \mathrm{~Hz}$. For oscillation at 0.5 $\mathrm{Hz}$, we calculated firing rate by counting the spikes in a 100 -msec-wide sliding window that was centered on the analysis bin. Although this analysis method causes some filtering of the data, it does not attenuate the responses to sinusoidal stimuli at frequencies below $1 \mathrm{~Hz}$. For oscillation of $4 \mathrm{~Hz}$, we avoided the low-pass filtering of the sliding window by calculating firing rate as the reciprocal of the interspike interval that contained the center of each bin. This method also filters the data, but only for frequencies in the range of the spontaneous firing rate of the unit spikes and not at frequencies as low as $4 \mathrm{~Hz}$. The averages were subjected to Fourier analysis with a fast Fourier transform to determine the fundamental components of head velocity and firing rate at each frequency. We calculated the sensitivity of each afferent to sinusoidal head rotation as the amplitude of the fundamental component of firing rate divided by the amplitude of the fundamental component of head velocity. The phase shift of each afferent's response was defined as the difference between the phase of the fundamental component of the firing rate and that of the sinusoidal head velocity signal.

The instantaneous firing rate during pulses of head velocity was computed by the algorithm of Lisberger and Pavelko (1986). An analog representation of the change in firing rate was obtained by aligning, on the onset of head motion, the responses to 50-100 pulses in the same direction. Head velocity and firing rate were then averaged at $1 \mathrm{msec}$ intervals for $200 \mathrm{msec}$ before and $300 \mathrm{msec}$ after the onset of the stimulus. We computed the firing rate at time $t, \operatorname{fr}(t)$, according to the algorithm

$$
\begin{aligned}
\operatorname{fr}(t) & =1 /\left(T_{i}-T_{i-1}\right) & & \text { if } t-T_{i}<T_{i}-T_{i-1}, \\
& =1 /\left(T_{i+1}-T_{i}\right) & & \text { if } t-T_{i} \geq T_{i}-T_{i-1},
\end{aligned}
$$

where $T_{i}$ represents the absolute time of occurrence of the $i$ th spike in the train and time $t$ falls between the $i$ th and $(i+1)$ th spike. This algorithm provides an accurate measure of the firing rate as long as the duration of the stimulus is long by comparison with the resting interspike interval (D. Broussard, C. deCharms, and S. Lisberger, unpublished obscrvations). This requirement was met in our data because the resting interspike intervals of vestibular afferents are short in comparison to the $50 \mathrm{msec}$ duration of the head acceleration during rapid changes in head velocity.

\section{Results}

Our goal was to use electrical stimulation to correlate the afferents activated at a given stimulation current with the properties of the eye movements evoked by the same current. In the first part of this article, we present the effect of stimulation current and of changes in the gain of the VOR on the eye movements evoked by electrical stimulation of the vestibular apparatus with single pulses and trains of pulses. In the second part, we describe relationships that reveal the physiological properties of the afferents activated at each stimulation current.

\section{Eye movements evoked by single electrical pulses when the gain of the $V O R$ is normal}

Figure $2 A$ shows averages of the eye velocity evoked by electrical stimulation of the superior semicircular canal over a range of currents. At each current, the response consisted of an initial rapid deflection of eye velocity away from the side of stimulation (contraversive, upward deflections in our records) and a later rebound in the ipsiversive direction (downward deflection of the traces). As the current was increased, the amplitude of the peak eye velocity increased, but there was no change either in the latency from the stimulus to the onset of eye movement (5 $\mathrm{msec})$ or in the latency to the first peak of eye velocity $(10 \mathrm{msec})$. Although it could be quite large in amplitude, the twitch of eye velocity was so short in duration that eye position (shown at the top of Fig. $2 A$ ) underwent a displacement of only $0.15^{\circ}$ at the highest stimulating current we used $(350 \mu \mathrm{A}$ in this monkey). For the two traces for stimulation at $75 \mu \mathrm{A}$ and $350 \mu \mathrm{A}$, the dashed lines show one SD of eye velocity, which was small on each experimental day. Electrical stimulation of the superior canal also evoked an upward component of eye velocity that we have not analyzed further.

Figure $2 B$ plots the peak eye velocity as a function of the stimulation current for the two monkeys we used to record vestibular afferents. Each point was obtained by averaging the peak eye velocity at one current for 3 consecutive days. The day-to-day consistency of the evoked eye velocity is demonstrated by the small error bars in Figure $2 B$, which show the SD of the mean peak eye velocity. The shape of the relationship between peak eye velocity and stimulation current was similar in the two monkeys. Peak eye velocity increased as a function of stimulation current up to a plateau at the highest currents. However, the data from two monkeys differed, both in the absolute value of eye velocity at the highest currents and in the ranges of current over which eye velocity rose steeply toward saturation.

To allow comparison of data among the two monkeys whose data appear in Figure $2 B$ and the other monkeys in this study, we used the relationship between eye velocity and current to express the stimulation current on a normalized scale. First, the peak eye velocities in Figure $2 B$ were normalized by expressing each value as a percentage of the maximum achieved at any current in that monkey. Then, the values of current for each monkey were scaled so that a value of 1 was assigned to the stimulation current that evoked $50 \%$ of the maximum eye velocity. The half-maximum value of eye velocity provides a reliable reference for normalizing along the current axis because it is on the steepest portion of the curve and thus is subject to minimal signal to noise error. The half-maximum values of stimulation current were $110.6 \mu \mathrm{A}$ and $292.9 \mu \mathrm{A}$ for monkeys $T$ and $U$, respectively. We cannot explain the difference in the scale of the current between the monkeys or the difference in the absolute value of the maximum evoked eye velocity, but we suspect it was partly due to minor differences in the placement of the stimulating electrode in the superior semicircular 

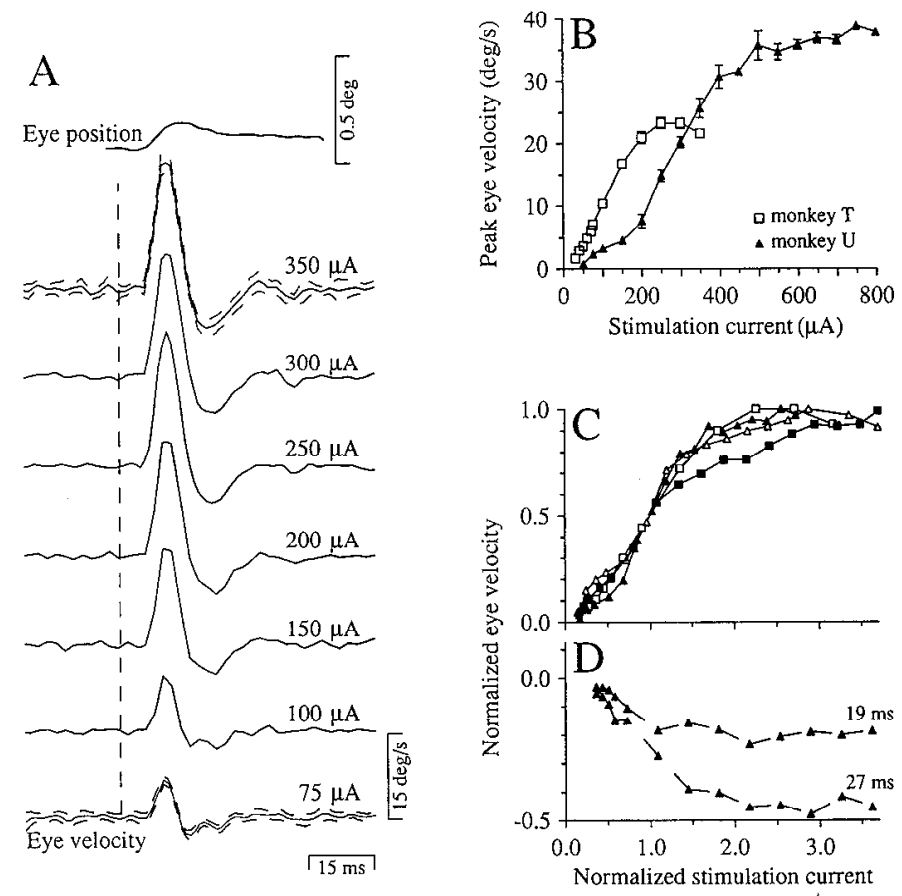

Figure 2. The horizontal eye movements evoked by stimulation of the superior semicircular canal with a single electrical pulse. $A$, Averages of horizontal eye velocity evoked by stimulation over a range of currents. The numbers on the right of each trace give the stimulation currents. The vertical dashed line shows the time of the stimulus. Dashed lines surrounding the top and bottom traces represent the SDs of eye velocity. The top trace shows the time course of average eye position for stimulation with a single pulse at $350 \mu \mathrm{A}$. $B$, The mean eye velocity at the peak of each response is plotted as a function of stimulation current. Open squares and solid triangles show data from monkeys $\mathrm{T}$ and $\mathrm{U}$, respectively. Error bars show SDs when they were larger than the size of the symbol. $C$, The data from $B$ are replotted and data from monkeys $\mathrm{R}$ (open triangles) and $\mathrm{Y}$ (solid squares) are plotted to show normalized peak eye velocity as a function of normalized stimulation current. $D$, Normalized eye velocity 19 and $27 \mathrm{msec}$ after the stimulus is plotted as a function of normalized stimulation current. The data in $D$ are from monkey $\mathrm{U}$.

canal and partly due to differences in the properties of the VOR pathways in different individuals.

Figure $2 C$ shows that the relationships between normalized peak eye velocity and normalized stimulation current were similar in the four monkeys included in this study. The curves for all four monkeys were nearly superimposed. Eye velocity rose steeply as normalized stimulation current was increased from 0 to 2.2 and saturated as normalized stimulation current was increased above 2.2. Only the data from monkey Y (solid squares) deviated slightly from the general pattern and showed some further increase in eye velocity as the normalized stimulation current was increased above 2.2. The similarity of the curves for the four monkeys provides evidence that our normalization procedure is an appropriate way to equate currents across monkeys and to compare directly the responses of different individuals.

The measurements of eye velocity in Figure 2, $B$ and $C$, were made at the peak of contraversive eye velocity, $10 \mathrm{msec}$ after the application of the electrical stimulus pulse. For one monkey, we also measured eye velocity during the ipsiversive rebound of eye velocity, 19 and $27 \mathrm{msec}$ after the stimulus pulse, and normalized the data according to the procedures outlined above. As a convention, we have plotted our graphs so that ipsiversive
A

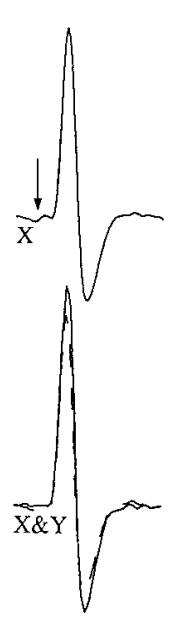

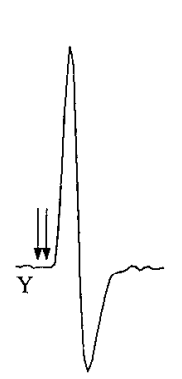

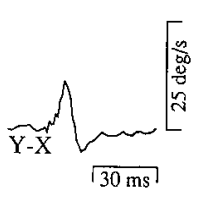

B

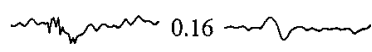

$\mathrm{C}$
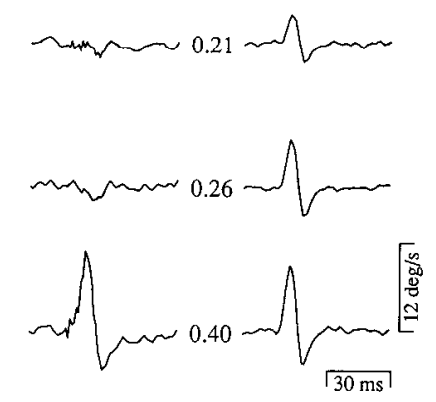

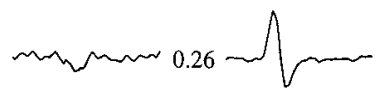

Figure 3. Eye movements evoked by the second of a double pulse stimulus. $A$, The trace labeled $X$ shows the average eye velocity produced by a single pulse at a normalized stimulation current of 3.16 . The trace labeled $Y$ shows the average cyc vclocity cvoked by two pulses at normalized currents of 3.16 and 0.4 and separated by 4 msec. The arrows show the times of stimulation. The two traces are superimposed in the traces labeled $X \& Y$, and the trace labeled " $Y$ - $X$ " shows the eye velocity evoked by double pulses $(Y)$ minus the eye velocity evoked by single pulses $(X) . B$, A series of eye velocity responses to the second of two pulses. The normalized stimulation current of the first pulse was 3.16. $C$, A series of eye velocity responses to asynchronous single pulses at the same currents. The numbers between the traces in $B$ and $C$ show the normalized stimulation current of the second pulse $(B)$ or the only pulse $(C)$.

eye velocity appears as a negative value of eye velocity. Figure $2 D$ shows that cyc velocity during the ipsiversive rebound became more negative as a function of current and, like the earlier peak eye velocity, reached a plateau at normalized stimulation currents above 2.2 .

Previous studies have shown that the threshold for the activation of primary afferents depends on the interval between the preceding spike in the afferent and the stimulus (Goldberg et al., 1984). The most reliable comparison of afferent thresholds and evoked eye movements requires that the electrical pulse occur at a fixed and known time after the last spike in the afferents activated by the stimulus. We therefore used a double pulse paradigm that was designed to synchronize the second pulse with the responses to the first pulse. The stimulation current for the first pulse was high enough to activate all the afferents and the second pulse always occurred $4 \mathrm{msec}$ later. 'This interval was $63 \%$ or less of the ISI of all the afferents in our sample. The stimulation current for the second pulse was varicd over the same range used to study the eye velocity responses to single, asynchronous pulses.

The averages of eye velocity in Figure $3 A$ illustrate the method used to extract the eye velocity response to the second pulse from the composite response to the double pulse stimulus. Trace $\mathrm{X}$ shows the eye velocity evoked by a single pulse at a normalized stimulation current of 3.16 , and trace $Y$ shows the eye velocity evoked by two consecutive pulses that were separated by $4 \mathrm{msec}$ and had normalized currents of 3.16 and 0.4 . These two traces were superimposed (X\&Y) and the response to the second shock $(\mathrm{Y}-\mathrm{X})$ was calculated by subtracting the eye velocity evoked by the first pulse $(\mathrm{X})$ from that evoked by the pair of pulses (Y). In Figure 3, $B$ and $C$ compare the eye velocity responses to the 


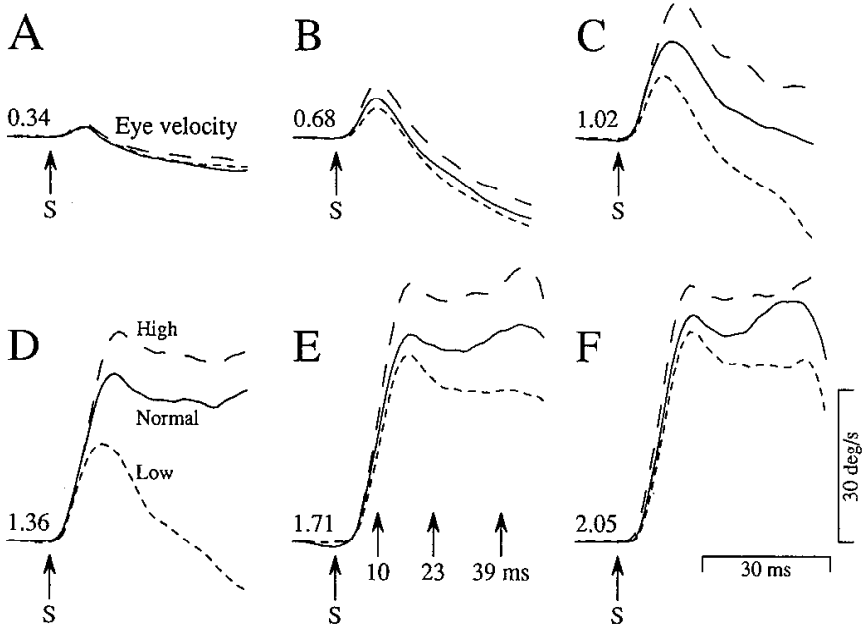

Figure 4. Effect of motor learning on the eye velocity evoked by trains of pulses applied to the superior semicircular canal in one monkey. Each trace shows averages of the responses to 12-16 trains. The short dash, solid, and long dash traces show averages of eye velocity when the gain of the VOR was low (0.32), normal (0.91), and high (1.54), respectively. The numbers on each trace give the normalized stimulation current. The arrows labeled $S$ indicate the time of onset of the train of pulses. The additional arrows in $E$ point to three specific times that were used in Figure 5 to analyze the data quantitatively. Data are from monkey U.

second pulse of a double pulse stimulus $(B)$ with the responses to a single pulse at each of four low values of normalized stimulation current $(C)$. When the second of a pair of pulses had normalized stimulation currents of $0.16,0.21$, and 0.26 , it did not evoke an eye velocity response ( $\Gamma$ ig. $3 B$ ), even though single pulses at the same currents evoked clear responses (Fig. $3 C$ ). When its normalized current was 0.4 , the second pulse evoked an eye velocity similar to that produced by single pulses at the same current. There was also a clear response to the second pulse when its normalized current was higher (not shown), but substantial facilitation in the eye velocity responses to double pulses made it impossible to compare quantitatively the relationship between peak eye velocity and stimulation current for single pulses with the relationship for the second of a pair of pulses.

\section{Effect of motor learning on eye movements evoked by single electrical pulses}

In a previous publication (Broussard et al., 1992), we documented the effect of changes in the gain of the VOR on the eye movements evoked by stimulation of the labyrinth with single electrical pulses. In the present study, we have repeated our earlier experiments using the normalization procedure to compare results across animals. Our results confirmed the earlier study. Briefly, changes in the gain of the VOR had a clear effect that was expressed in the earliest part of the eye movement evoked by electrical stimulation with single pulses. The effect of changes in the gain of the VOR grew as a function of stimulation current and as a function of time after the stimulus. The slope of the relationship between the evoked eye velocity at a given current and the gain of the VOR was largest during the ipsiversive rebound in eye velocity.

Effect of motor learning on eye movements evoked by trains of electrical stimuli

Figure 4 illustrates the eye movements evoked in one monkey by trains of 15 pulses at a frequency of $200 \mathrm{~Hz}$ over a range of stimulation currents when the gain of the VOR was low (short dashes), normal (solid traces), or high (long dashes). Each trace shows the average eye velocity for $10 \mathrm{msec}$ before and $46 \mathrm{msec}$ after the onset of these stimuli. The train of stimulus pulses began at the arrows labeled " $S$ " and continued throughout the records that are illustrated. We did not show the full $75 \mathrm{msec}$ of the response because the smooth eye movements in the last $30 \mathrm{msec}$ of the train were often contaminated with reflexive saccades, especially for stimulation with high currents. The latency from the onset of the stimulus train to the onset of the evoked eye velocity was the same as the latency measured for single pulses ( $5 \mathrm{msec}$ )

When the gain of the VOR was normal (solid traces), trains of stimuli at different currents evoked eye velocities with very different profiles. When the normalized stimulation current was 0.34 (Fig. $4 A$ ) or lower, trains of electrical pulses evoked only tiny eye movements. When the normalized stimulation current was 0.68 (Fig. $4 B$ ), the initial deflection in eye velocity moved the eyes contraversive to the labyrinth being stimulated. However, the eye velocity decayed quickly, crossed zero, and reversed direction, causing the eyes to move toward the side of stimulation at significant speeds. The reversal in the direction of eye movement also appeared in the eye position traces (not shown) and therefore could not have been an artifact introduced by the analog differentiator used to obtain eye velocity. At a normalized stimulation current of 1.02 (Fig. 4C), a train of stimuli caused eye velocity to increase rapidly to a peak in the contraversive direction and then to decay back to zero within about $50 \mathrm{msec}$ after the onset of the stimulus. When the gain of the VOR was normal, trains of electrical pulses evoked eye velocity that was contraversive throughout the response only if the normalized stimulation current was equal to or greater than 1.3. In Figure $4 D-F$, eye velocity showed a rapid initial rise and was sustained throughout the stimulus train. Measurements of initial eye acceleration showed that the rate of rise of eye velocity saturated at a normalized stimulation current of 1.71 (Fig. 4E), but the level of sustained eye velocity continued to increase when the normalized stimulation current was increased to 2.05 (Fig. $4 F$ ).

The general trends illustrated in Figure 4 are representative of the three monkeys ( $R, U$, and $Y$ ) studied with trains of pulses at varying currents when the gain of the VOR was normal (monkey $T$ was studied with trains of pulses at only one current). The initial rise in eye velocity was similar in all monkeys and the sustained eye velocity was contraversive only for higher currents. Different monkeys showed slightly different trajectories of eye velocity in the sustained part of the response and differed in the exact value of current that served as the border between responses like those in Figure $4, B$ and $C$, and responses like those in Figure $4 D-F$. Finally, statistical analysis showed that the average records in Figure 4 provide good estimates of the individual responses to trains of pulses. The standard deviations of eye velocity (not shown) were less than $2 \%$ sec.

The effect of changes in the gain of the VOR on the eye movements evoked by trains of electrical pulses depended on the stimulation current. When the normalized stimulation current was 0.34 (Fig. $4 A$ ), neither the amplitude nor the trajectory of the tiny eye velocity responses depended clearly on the gain of the VOR. At a normalized stimulation current of 0.68 (Fig. $4 B$ ), changes in the gain of the VOR had a small effect on the eye velocity evoked by trains of electrical stimuli. The eye velocity evoked when the gain of the VOR was high (long dashes) 

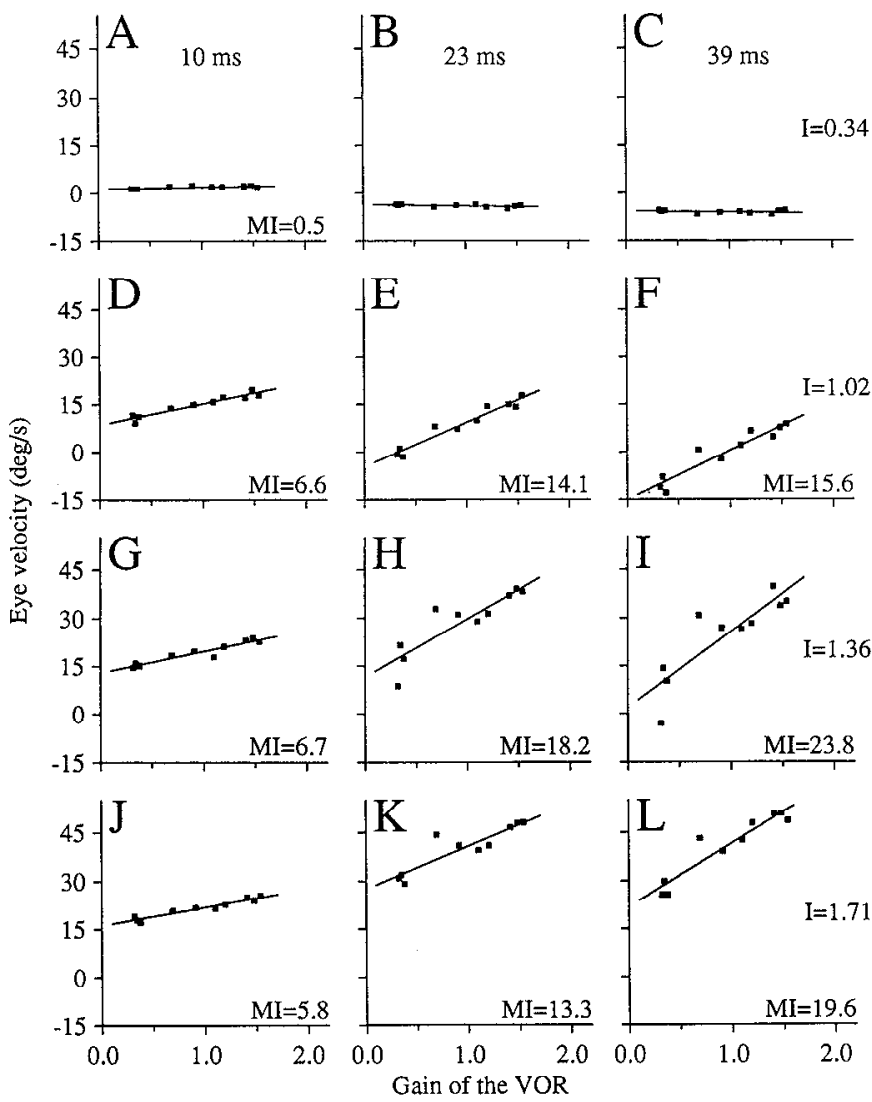

Figure 5. Relationship between the evoked eye velocity and the gain of the VOR at three times after the onset of trains of electrical pulses. Each graph plots data obtained on multiple days at a given normalized stimulation current and a single latency after the onset of the stimulus. Each column of graphs displays the relationship at one time after the onset of stimuli at four different currents. Each row of graphs displays the relationship at one stimulation current and at three times after the onset of the stimulus. The normalized stimulation current $(I)$ is given by the numbers to the right of each row. The lines in each graph were obtained by linear regression. The numbers in the lower right corner give the slopes of the lines, whenever the slope was significantly different from zero $(F$ test, $p<0.05)$. The slope of each line estimates the degree of learning in the VOR and is called the modification index $(M I)$ : Data are from monkey $U$.

or low (short dashes) differed from that evoked when the gain of the VOR was normal (solid lines) by a small constant amount throughout almost the entire duration of the eye movement. At higher stimulation currents (Fig. $4 C-F$ ), changes in the gain of the VOR had more dramatic effects on the eye movements evoked by trains of electrical stimuli. In each case, the differences in the initial rising phase of the response were relatively small and the largest differences appeared later in the train. The most striking effects appeared at normalized stimulation currents of 1.02 and 1.36. For these currents, the eye velocity was contraversive throughout the part of the response that is shown when the gain of the VOR was normal or high. When the gain of the VOR was low, however, the eye velocity showed an initial contraversive movement and then decayed so that eye velocity was ipsiversive in the later part of the train. At the highest normalized stimulation currents of 1.71 and 2.05 (Fig. $4 E, F$ ), the amplitude but not the direction of the response depended on the gain of the VOR; thus, eye velocity was contraversive throughout the response, even when the gain of the VOR was low.
A
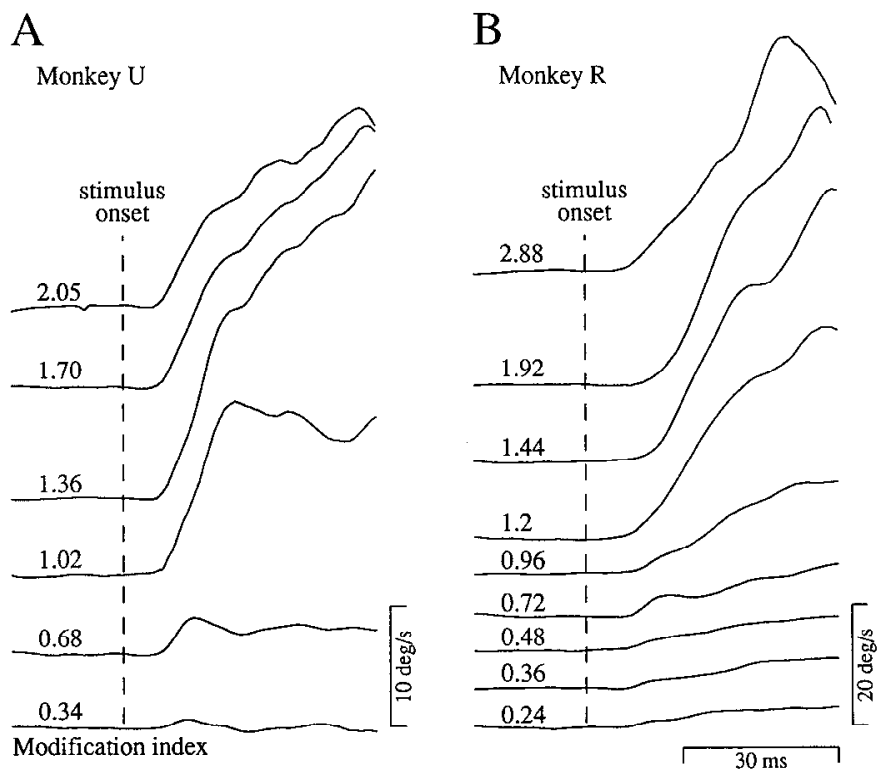

Figure 6. Time course of the MI for two monkeys. The dashed line labeled stimulus onset shows the time of the onset of the train of pulses. The numbers at the left of each trace give the normalized stimulation current. Because the eye velocity and the MI evoked by trains of pulses were smaller at each stimulation current in monkey $\mathrm{U}$, his data are shown at twice the vertical amplification as those from monkey $\mathrm{R}$.

To quantify the expression of learning at different stimulation currents and at different latencies during the response, we measured the value of the averaged eye velocity evoked by each train of pulses at latencies of 10,23 , and $39 \mathrm{msec}$ after the onset of the stimulus. Arrows in Figure $4 E$ point to these times along the course of the eye movement. We then made plots of eye velocity at each time as a function of the gain of the VOR. In Figure 5, for example, each graph summarizes the effect of motor learning on eye velocity for one stimulation current at one latency after the onset of the stimulus. Each row of graphs shows data for one stimulation current measured at three different latencies after the onset of the stimulus, and each column shows data measured at one latency for four different currents. The slope of the line in each graph, computed by linear regression, provides a quantitative estimate of the effect of VOR gain on eye velocity and has been called the "modification index" (MI) by Broussard et al. (1992). MI was essentially zero in the first row of graphs and was statistically different from zero only in Figure $5 A$ ( $F$ test, $p<0.05$ ). Thus, changes in the gain of the VOR did not have a large effect on the eye velocity evoked by trains of stimuli for small values of normalized stimulation current (Fig. 54-C). For the other nine graphs (Fig. $5 D-L$ ), the slope of the linear regression line was significantly different from zero and MI is given in the lower right corner of each graph. For normalized stimulation strengths of $1.02,1.36$, and 1.71, MI was always smaller $10 \mathrm{msec}$ after the onset of the stimulus than 23 or $39 \mathrm{msec}$ after the onset of the stimulus. For a normalized stimulation current of 1.02 , MI was similar at 23 and $39 \mathrm{msec}$ after the onset of the stimulus (Fig. $5 E, F$ ). At higher currents, however, MI was about 1.5 times as large $39 \mathrm{msec}$ after the onset of the stimulus (Fig. $5 I, L$ ) as it was $23 \mathrm{msec}$ after the onset of the stimulus (Fig. $5 H, K$ ). Although these data show that the effect of changes in the gain of the VOR is most pronounced later in the eye movements evoked by electrical stimulation of the vestibular apparatus, they do not contradict our 


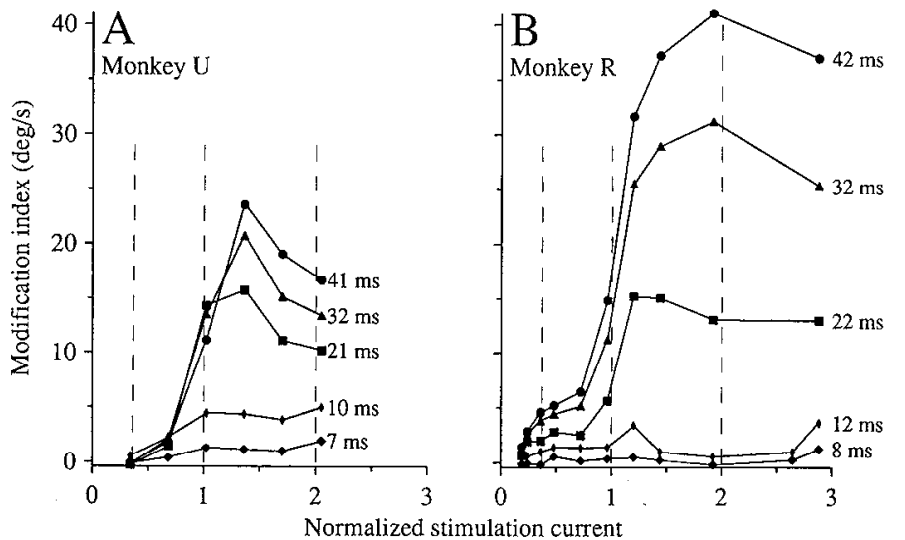

Figure 7. Relationship between MI and normalized stimulation current at different latencies after the onset of stimulation for two monkeys. Each point was measured from the data in Figure 6 and the points measured at the same time after the onset of the stimulus were connected. The numbers on the right of the graphs give the times when the measurements were made. The vertical dashed lines show normalized stimulation currents of $0.37,1.0$, and 2.0 , respectively. MI is shown on the same scale for the two monkeys

earlier finding that changes in the gain of the VOR affect the earliest part of the eye movements evoked by electrical stimuli (Broussard et al., 1992). At normalized stimulation currents equal to or greater than 0.34 , the relationship between eye velocity $10 \mathrm{msec}$ after the onset of the stimulus and the gain of the VOR (e.g., Fig. $5 A, D, G, J$ ) always had a significant positive slope.

Figure 5 shows snapshots of the effect of motor learning on the eye movements evoked by trains of pulses at three specific moments after the onset of the stimulus. To visualize the time course of the effect of VOR gain on eye velocity and analyze the data more thoroughly, we calculated MI for each stimulation current and at each millisecond during the response and we plotted the time course of MI for each stimulation current (Fig. 6). In Figure 6, each of the curves for a given monkey is plotted on the same vertical scale, but the scale differs by a factor of two for the two monkeys and the baselines for the curves are shifted progressively upward as current increases. Each trace establishes its own baseline because MI was always close to zero in the period before stimulus onset (vertical dashed lines).

The effect of changes in the gain of the VOR was similar in monkeys $\mathrm{U}$ and $\mathrm{R}$ (Fig. $6 A, B$ ). We were able to obtain quality data in this experiment for only these two of our four monkeys. Monkey $\mathrm{T}$ was the first monkey we studied and we used him to evaluate the effect of changes in the gain of the VOR on the eye movements evoked by trains of pulses at only one current. In monkey $\mathrm{Y}$ we were unable to obtain a full set of data because he did not fixate well during the application of trains of pulses at high currents. However, the fragmentary results we obtained on monkeys $\mathrm{T}$ and $\mathrm{Y}$ were entirely consistent with those illustrated in Figure 6. In monkeys $\mathrm{R}$ and $\mathrm{U}$, MI remained small throughout the duration of the evoked eye velocity when the normalized stimulation current was 0.72 or below. MI grew rapidly as normalized stimulation current was increased from 0.72 to 1.44 and showed little or no further growth as the stimulation current was increased further. The time course of MI at different stimulation currents showed that the time of the largest effect of motor learning depended on the value of normalized stimulation current. At normalized stimulation currents be- tween 0.48 and 0.72 , almost all of the increase in MI occurred early in the response. At normalized stimulation currents above 1.2 , in contrast, MI increascd throughout the response, indicating that the effect of VOR gain was even more pronounced at longer latencies after the stimulus.

Figure 7 provides an analysis of how MI varied as a function of normalized stimulation current at five different times from 8 to $42 \mathrm{msec}$ after the onset of the stimulus. To obtain Figure 7, we measured MI at these five times from the traces in Figure 6 , plotted MI as a function of the normalized stimulation current, and connected the points measured at each individual time. In both monkey $U$ (Fig. $7 A$ ) and monkey $\mathrm{R}$ (Fig. $7 B$ ), MI was uniformly positive but was small in the interval from 8 to 12 msec after the onset of the stimulus. Thus, changes in the gain of the VOR had a small effect on the earliest component of the eye velocity evoked by a train of pulses, even at the highest values of normalized stimulation current. At later times, MI showed a strong relationship to stimulation current. MI was relatively small when the normalized stimulation current was less than 0.7 , showed a dramatic increase as the normalized stimulation current was increased from 0.7 to 1.5 , and declined slightly at higher values of normalized stimulation current. The largest value of MI occurred 41-42 msec after the onset of the stimulus at normalized stimulation currents of 1.36 and 1.71 in monkeys $\mathrm{U}$ (Fig. $7 A$ ) and $\mathrm{R}$ (Fig. $7 B$ ).

\section{Resting discharge properties of primary afferents}

We obtained detailed information about the responses of 110 vestibular primary afferents that showed increased firing for ipsiversive angular head rotation and therefore were identified as afferents from the horizontal canal. The spontaneous firing rate of horizontal canal afferents ranged from 29 to 158 spikes/ sec and had the distribution shown in Figure $8 A$. The distribution was similar to that in previous populations of afferents recorded in awake rhesus monkeys (Miles and Braitman, 1980). The normalized coefficient of variation ( $\mathrm{CV}^{*}$ ) ranged from 0.02 to 0.61 , and its distribution (Fig. $8 B$ ) was similar to that in an earlier study of vestibular afferents in squirrel monkeys (Goldberg et al., 1984). We used the criteria of Goldberg et al. (1984) to classify the spontaneous discharge of afferents. Individual afferents were classified as regular if $\mathrm{CV}^{*}$ was less than $0.1(61 \%$ of our sample), as intermediate if $\mathrm{CV}^{*}$ was between 0.1 and 0.2 $(29 \%)$, and as irregular if $\mathrm{CV}^{*}$ was greater than $0.2(10 \%)$.

\section{Threshold of afferents for electrical stimulation}

Figure $9 A-C$ contains peristimulus histograms that illustrate the responses of one horizontal canal afferent to single electrical pulses at three currents surrounding the threshold for activating the fiber. Each histogram was obtained by summing the spikes in $0.1 \mathrm{msec}$ bins for $20 \mathrm{msec}$ before and $80 \mathrm{msec}$ after each of approximately 100 pulses to the labyrinth; only the relevant part of each histogram is illustrated in Figure 9. In each histogram, the tall, narrow peak labeled " $T$ " contains the naturally occurring action potentials that served as the trigger for each electrical pulse and therefore occurred at a fixed time before the stimulus. The number of events recorded in this peak equal the number of traces used to generate each histogram. When the stimulation current was $250 \mu \mathrm{A}$ (Fig. 9A), the afferent was activated only rarely. Instead the afferent usually maintained its regular spontaneous firing rate, such that most of the spikes fell in the peaks labeled " 1 " and "3." The width of peaks 1 and 3 reflects the slight variation in the ISIs seen even in the afferents 


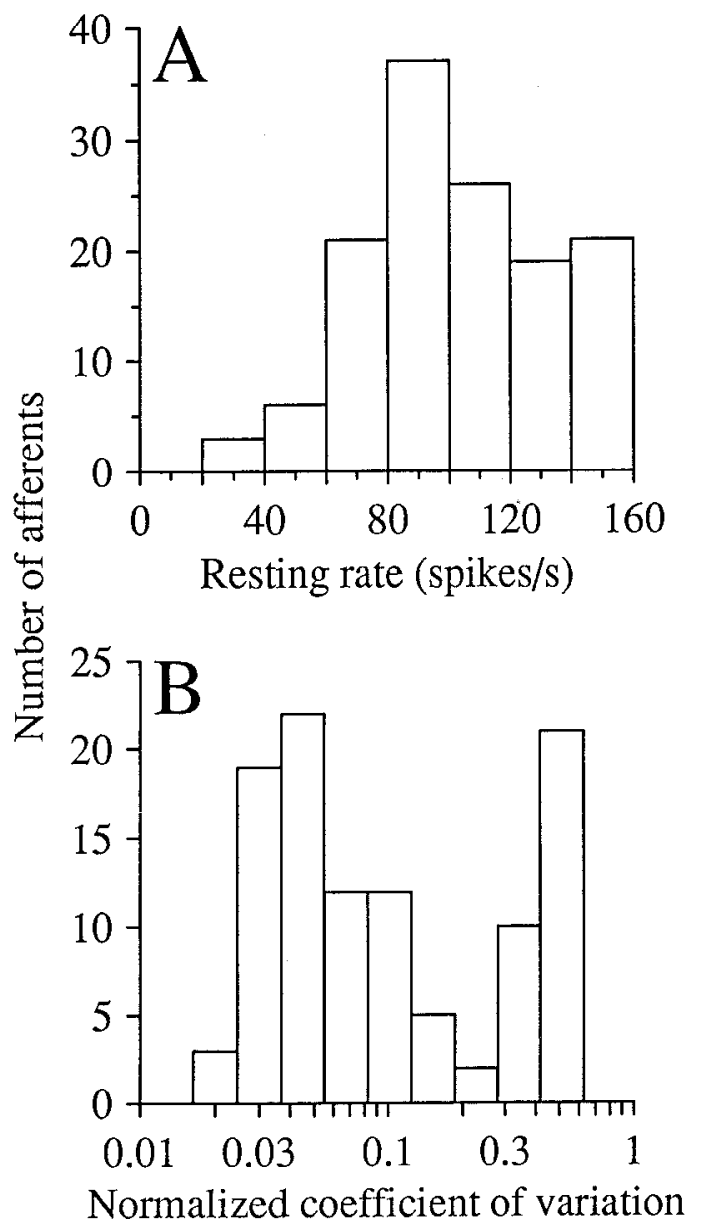

Figure 8. Distribution of resting rate $(A)$ and normalized coefficient of variation or $\mathrm{CV}^{*}(B)$ for the horizontal semicircular canal afferents in our study. The histogram in $B$ was created by first converting the distribution of $\mathrm{CV}^{*}$ to a logarithmic scale and then accumulating the numbers of afferents in bins that had equal widths on the logarithmic scale.

with the most regular spontaneous firing. On the occasional times when the stimulus activated the afferent, the evoked spikes fell in the small narrow peak labeled "E."

When the stimulation current was $270 \mu \mathrm{A}$ (Fig. 9C), the fiber was activated almost every time such that the height of the peak labeled " $E$ " (evoked spike) approached that of the peak labeled " $T$ " (trigger spike). Activation of the fiber reset the impulse generator such that the next spike was delayed and occurred in peak 2 instead of in peak 1 . The interval between the evoked spike and its successor was $0.7-1.0 \mathrm{msec}$ longer than the mean interval between spontaneous spikes but subsequent ISIs were the same duration as the fiber's spontaneous intervals. On the few times that the fiber was not activated, it followed its natural firing rhythm and the spikes that followed the stimulus accumulated in the small peaks labeled " 1 " and " 3 ." When the stimulation current was $260 \mu \mathrm{A}$ (Fig. $9 \mathrm{~B}$ ), the fiber was activated about half the time. If the fiber was activated, then the evoked spikes fell in the peak labeled "E" and the subsequent spikes fell in peak 2, which again followed the evoked spike by an ISI that was slightly longer than the mean measured during spontaneous firing. If the fiber was not activated, then the spikes that followed the stimulus occurred at the regular intervals and fell in peaks 1 and 3 .
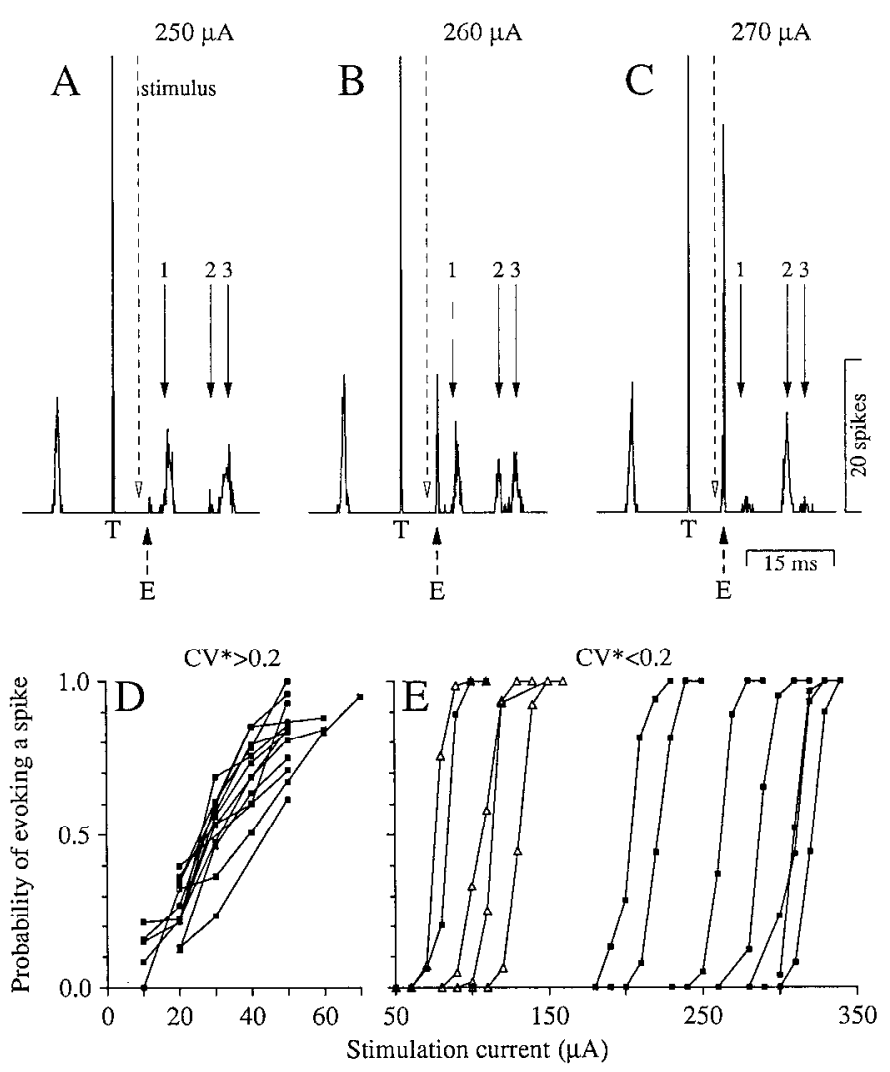

Figure 9. Relationship between the probability of evoking a spike and stimulation current for vestibular primary afferents. $A-C$, Peristimulus histograms used to determine the threshold for electrical activation of an afferent with single pulses. Each histogram shows the response of the same afferent to 100 pulses at currents of $250 \mu \mathrm{A}(A), 260 \mu \mathrm{A}(B)$, and $270 \mu \mathrm{A}(C)$. The vertical dashed line shows the time of the stimulus, which was always triggered off a naturally occurring spike in the afferent under study. The trigger spikes accumulated in the responses labeled $T$ and spikes evoked by the stimulus accumulated in the peak labeled $E$. The peaks labeled $I, 2$, and 3 contain the spikes that occurred one ISI after the trigger spikes, one ISI after the evoked spikes, or two ISIs after the trigger spikes, respectively. $D$, Curves showing the probability of activation as a function of stimulation current for a selection of afferents with irregular spontaneous discharge $\left(\mathrm{CV}^{*}>0.2\right)$ in monkey T. $E$, Curves showing the probability of activation as a function of stimulation current for a selection of afferents with intermediate spontaneous discharge (open triangles, $0.1<\mathrm{CV}^{*}<0.2$ ) and rcgular spontancous discharge (solid symbols, $\mathrm{CV}^{*}<0.1$ ). In $D$ and $E$, each set of connected points shows data from an individual afferent.

In Figure 9, $D$ and $E$ summarize the relationship between the probability of activation and the stimulation current for a sample of the afferents recorded from monkey $T$; the data from monkey U were similar. We used histograms like those in Figure $9 A-C$ to calculate the probability of activating an afferent as the ratio of the number of events in the evoked spike peak to the number of stimuli applied. Each set of connected points shows data from a different afferent. The afferents with irregular spontaneous discharge (Fig. 9D) all showed similar activation curves. None of the afferents were activated for stimuli below $20 \mu \mathrm{A}$ and all were activated by nearly every electrical pulse when the current was $60 \mu \mathrm{A}$ or above. The activation curves for afferents with intermediate spontaneous discharge (open symbols in Fig. $9 E$ ) and regular spontaneous discharge (solid symbols in Fig. $9 E$ ) each had the same general shape, but the curves from individual afferents were distributed across a wide range of cur- 


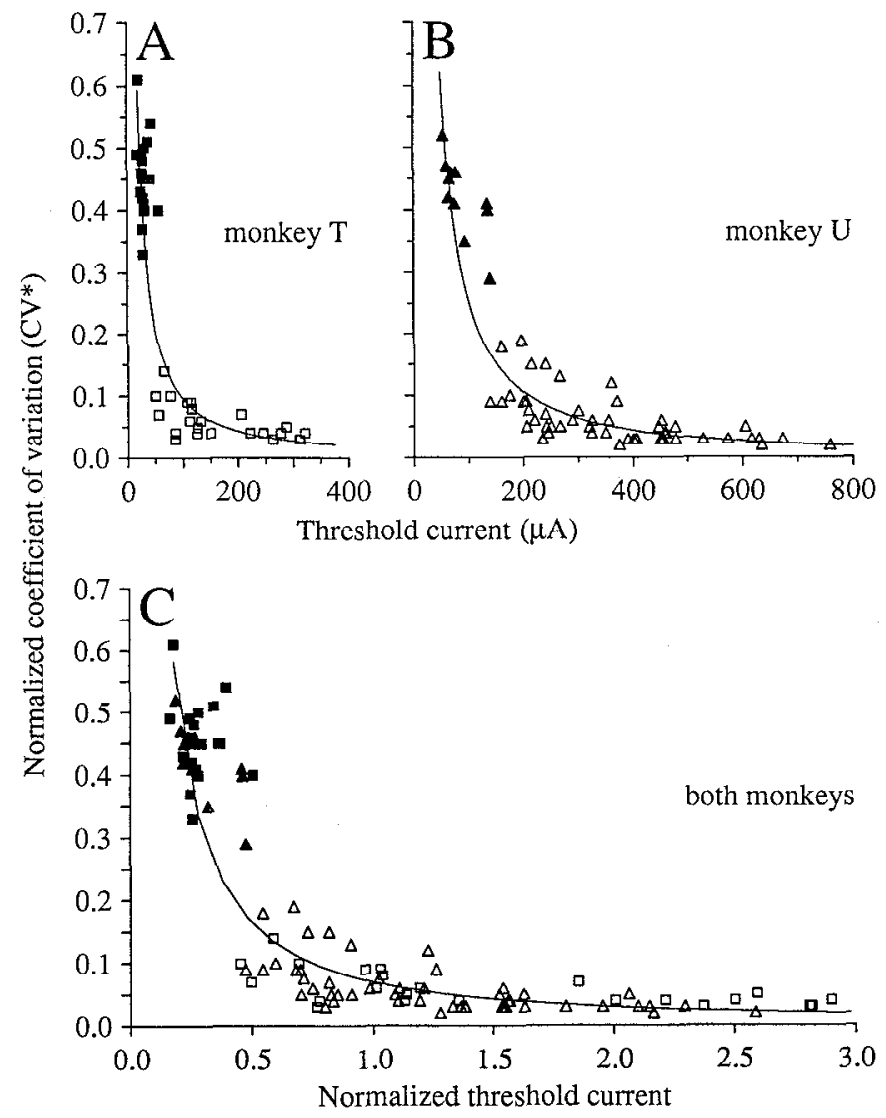

Figure 10. Relationship between discharge regularity and threshold current for vestibular primary afferents. $A$ and $B$, Normalized coefficient of variation $\left(\mathrm{CV}^{*}\right)$ is plotted as a function of the actual threshold current for all afferents from monkey $\mathrm{T}(A)$ and monkey $U(B)$. $C$, The data from monkeys $\mathrm{T}$ and $\mathrm{U}$ are pooled and $\mathrm{CV}^{*}$ is plotted as a function of normalized threshold current. The solid symbols show data for afferents with irregular spontaneous discharge and the open symbols show data for afferents with regular or intermediate spontaneous discharge. The curves represent the power law function that provided the best fit to the data in each graph (see Results for equations).

rents. Each activation curve showed a rapid rise that took the probability of evoking a spike from less than 0.1 to nearly 1 over a current range of 20-30 $\mu \mathrm{A}$. We calculated the threshold for activation for each afferent by interpolating along each activation curve to find the current at which the probability of evoking a spike would have been 0.5 .

\section{Relationship between the threshold for electrical activation and the physiological properties of afferents}

There was a clear relationship between the discharge regularity (measured by $\mathrm{CV}^{*}$ ) and the threshold of each afferent for electrical stimulation. The relationship is summarized separately for the two monkeys in Figure $10, A$ and $B$, where each point represents data from one afferent. Although the samples recorded in the two monkeys show very similar distributions of $\mathrm{CV}^{*}$, quite different ranges of current were required to activate a given set of afferents in the two monkeys. Afferents with irregular spontaneous discharge (solid symbols) had the lowest thresholds, between 18 and $56 \mu \mathrm{A}$ in monkey $\mathrm{T}$ (Fig. 10A) and between 55 and $140 \mu \Lambda$ in monkey $U$ (Fig. 10B). $\Lambda$ fferents with intermediate and regular spontaneous discharge (open symbols) had thresholds between 60 and $321 \mu \mathrm{A}$ in monkey $\mathrm{T}$ (Fig. 10A) and between 139 and $760 \mu \mathrm{A}$ in monkey $\mathrm{U}$ (Fig. 10B). Among the intermediate and regular afferents, there was a tendency for those with higher values of $\mathrm{CV}^{*}$ to be recruited at lower currents and for those with lower values of $\mathrm{CV}^{*}$ to be recruited at higher currents. All afferents that showed increased firing for ipsiversive head motion were activated at some current by electrical stimulation. Linear, logarithmic, exponential, and power law regressions were performed on the data in Figure 10. The curve that provided the best fit in each graph was obtained with power law regression $\mathrm{CV}^{*}=a T^{-h}$, where $T$ represents the threshold current for activation of each afferent. The correlation coefficients were statistically significant in each monkey $(r=0.89, p$ $<0.01$ for monkey T; $r=0.93, p<0.01$ for monkey $U$ ) and the value of the exponent $(b)$ was similar in the two monkeys ( $b=1.137$ for monkey $\mathrm{T}, b=1.280$ for monkey $\mathrm{U}$ ). However, the different ranges of current required to activate the afferents in the two monkeys was responsible for a large difference in the value of $a$, which was 18.81 in monkey $T$ and 92.89 in monkey $\mathbf{U}$.

The differences between the two monkeys in the relationship between $\mathrm{CV}^{*}$ and threshold current were eliminated when we used the procedure outlined in Figure 2 to normalize the current according to the eye movements evoked by single pulses to the vestibular apparatus. Figure $10 \mathrm{C}$ plots $\mathrm{CV}^{*}$ as a function of the normalized threshold current for the full samples of afferents from monkey $\mathrm{T}$ (squares) and monkey $\mathrm{U}$ (triangles). For both monkeys, all of the afferents recruited at a normalized stimulation current below 0.4 showed irregular spontaneous firing. The afferents recruited at normalized stimulation currents between 0.4 and 1.3 could have irregular, intermediate, or regular spontaneous discharge. The afferents recruited at normalized threshold currents above 1.3 all had regular spontaneous discharge and included the afferents with the lowest values of CV*. Power law regression of the relationship between $\mathrm{CV}^{*}$ and normalized threshold current for each monkey now yielded similar values for all the coefficients of the equation: $a=0.085, b=$ $1.137, r=0.81$ for monkey $\mathrm{T}$, and $a=0.064, b=1.294, r=$ 0.93 for monkey $U$. This validated our normalization procedure in terms of the response properties of primary afferents and demonstrated that it was legitimate to group data across monkeys in the experiments presented both earlier and later in this article.

Figure 11 combines the data from the two monkeys and analyzes the relationship between the responses to sinusoidal head rotation and the normalized threshold current for electrical stimulation. In Figure $11, A$ and $C$ plot the sensitivity to head velocity and the phase shift between firing rate and head velocity as a function of the normalized threshold current for each afferent studied during sinusoidal rotation at $0.5 \mathrm{~Hz}, B$ and $D$ plot the same information for sinusoidal head rotation at $4 \mathrm{~Hz}$. In Figure $11, A$ and $B$, one group of afferents (open squares and plus signs) formed a continuum and showed a relationship between the sensitivity to head velocity and normalized threshold current. In this group, afferents with thresholds at normalized currents less than 0.6 had high values of sensitivity. Sensitivity to head velocity for sinusoidal head turns was inversely correlated with normalized threshold current for electrical stimuli, so that afferents with thresholds between 0.6 and 1.3 had a wide range of sensitivity to head velocity and those with thresholds above 1.3 had the lowest sensitivity of the group. $\Lambda$ second group of afferents (solid squares) had very low thresholds for electrical stimulation but did not appear to be continuous with 

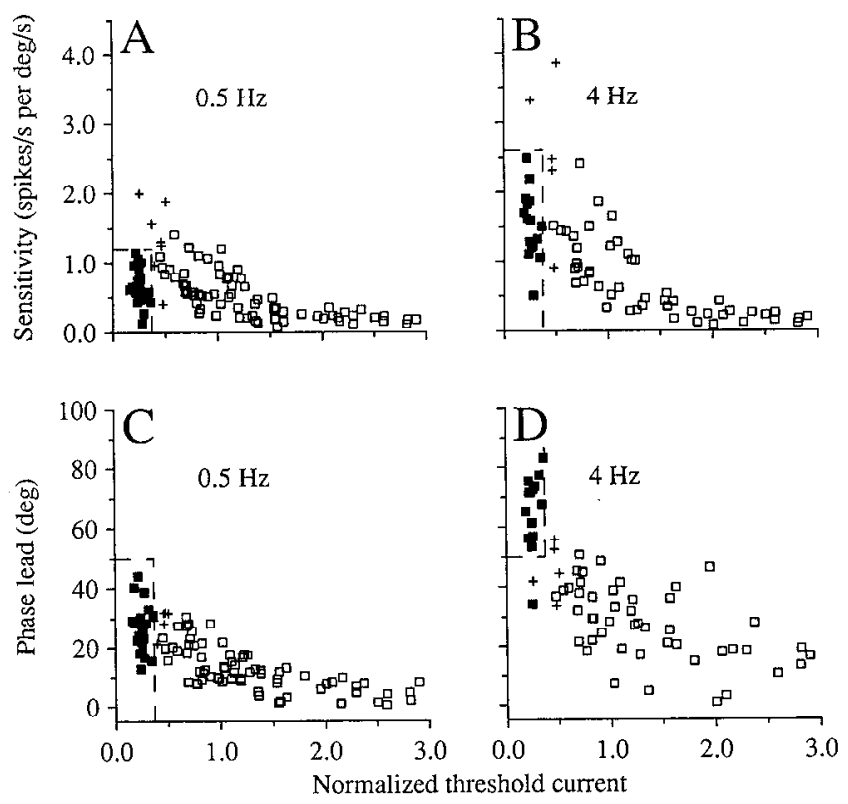

Figure 11. Relationship between afferent response properties for sinusoidal vestibular stimulation and normalized threshold current for electrical stimulation. Each graph combines data from monkeys $\mathrm{T}$ and $\mathrm{U}$ and plots one measure of responses to natural stimuli as a function of normalized threshold current. $A$, Sensitivity to head velocity at 0.5 $\mathrm{Hz}, \pm 10^{\circ} . B$, Sensitivity to head velocity at $4 \mathrm{~Hz}, \pm 1.25^{\circ}$. C, Phase shift between firing rate and head velocity at $0.5 \mathrm{~Hz}$. $D$, Phase shift between firing rate and head velocity at $4 \mathrm{~Hz}$. Positive values of phase shift indicate that firing rate led head velocity. Open symbols show data from afferents with intermediate and regular spontaneous discharge. Solid symbols show data from afferents that had irregular spontaneous discharge and, based on statistical analysis (see Results and Table 1), did not fit with the rest of the sample. Plus signs show data from afferents with irregular spontaneous discharge that, based on the same statistical analysis, had sensitivities to head velocity that fit with the intermediate and regular afferents.

the rest of the population because they had low values of sensitivity to head velocity.

Statistical analysis of the sensitivity to head velocity confirmed that the afferents formed two separable groups for rotation at 0.5 and $4 \mathrm{~Hz}$ (see Table 1). If we treated the entire sample of afferents as a single group, then the relationship between sensitivity to head velocity and normalized threshold current was fit poorly by power law regression. If we performed the same analysis for only the afferents with regular and intermediate discharge regularity (open squares in Fig. $11 \mathrm{~A}$ ), then the fit was improved significantly. However, we obtained the best correlation between sensitivity to head velocity and normalized threshold for electrical stimulation if the analysis included all the afferents except those represented by solid squares. A normalized threshold current of 0.37 provided an almost perfect dividing line between the afferents that did fit (open squares and plus signs) and did not fit (solid squares) on a power law relationship between sensitivity to head velocity and normalized threshold current. Therefore, we will use a normalized threshold current of 0.37 as a dividing point when we wish to refer to a group of afferents with highly irregular spontaneous discharge and low sensitivity to head velocity.

Statistical analysis revealed that the entire sample of afferents formed a continuous relationship between the phase with respect to head velocity at $0.5 \mathrm{~Hz}$ (Fig. 11C) and $4 \mathrm{~Hz}$ (Fig. 11D) and the normalized threshold current for electrical stimulation. Log-
Table 1. Statistical analysis of the relationship between afferent responses to sinusoidal rotation and threshold for electrical stimulation

\begin{tabular}{lcccccc} 
& $a$ & $b$ & $r_{s}$ & $c$ & $d$ & $r_{\mathrm{ph}}$ \\
\hline $0.5 \mathrm{~Hz}$ & & & & & & \\
$\quad$ All afferents & 0.41 & 0.54 & 0.47 & 14.23 & 23.01 & 0.80 \\
CV* $<0.2$ & 0.48 & 1.10 & 0.70 & 14.35 & 25.91 & 0.73 \\
$T>0.37$ & 0.48 & 1.08 & 0.81 & 14.82 & 28.25 & 0.79 \\
$4.0 \mathrm{~Hz}$ & & & & & & \\
All afferents & 0.58 & 0.95 & 0.60 & 32.15 & 46.96 & 0.82 \\
CV*<0.2 & 0.69 & 1.48 & 0.74 & 29.96 & 34.29 & 0.58 \\
$T>0.37$ & 0.69 & 1.46 & 0.87 & 30.26 & 35.20 & 0.65
\end{tabular}

The regression equation for sensitivity to head velocity (s) was $s=a T^{-\mathrm{h}}$, where $T$ is normalized threshold current. The regression equation for phase shift (ph) was ph $=c-d \times \ln (T)$. The values $r_{s}$ and $r_{\mathrm{ph}}$ give the correlation coefficients for the sensitivity and phase regressions, respectively. The other columns give the values of the parameters. Afferents were pooled for the two monkeys.

arithmic regression provided the best fit for the relationship between phase lead and threshold (Table 1, 4.0 $\mathrm{Hz}$ data) and was performed separately for the afferents with intermediate and regular spontaneous discharge (open squares), for all afferents shown by open squares and plus signs, and for the whole sample of afferents. For stimulation at both 0.5 and $4 \mathrm{~Hz}$, the correlation coefficient was highest when all the afferents were considered as one group. In general, the phase difference between afferent firing rate and head velocity was larger for head oscillation at $4 \mathrm{~Hz}$ than for oscillation at $0.5 \mathrm{~Hz}$. The increase in phase lead was most pronounced for the afferents with normalized threshold currents below 0.37 , some of which showed firing at $4 \mathrm{~Hz}$ more closely related to head acceleration than to head velocity.

We next evaluated the relationship between the threshold for electrical activation of 38 afferents and the properties of their responses to rapid changes in head velocity. Figure $12 \mathrm{~A}$ shows the head velocity stimulus and the averaged firing rates of two afferents that responded to electrical stimulation at normalized threshold currents of 0.25 and 2.3. The stimulus provided a rapid head acceleration for $50 \mathrm{msec}$ followed by sustained head motion at $30 \% \mathrm{sec}$. For the afferent with a normalized threshold of 0.25 (top trace in Fig. 12A), the averaged firing rate began to increase $6 \mathrm{msec}$ after the onset of head acceleration, reached a peak of 106 spikes/sec above the resting rate $11 \mathrm{msec}$ later, and then dropped sharply to a steady firing rate only 45 spikes/sec above resting rate. For the afferent with a normalized threshold current of 2.3 (middle trace in Fig. 12A), the average firing rate began to increase $10 \mathrm{msec}$ after the onset of head acceleration and rose gradually, with little or no overshoot, to a steady firing rate that was 15 spikes/sec above resting rate.

We quantified the degree of overshoot in the firing rate of afferents during rapid changes in head velocity by computing the dynamic index (DI), which is defined as the ratio of the peak change in firing rate ( $\mathrm{X}$ in Fig. $12 A$ ) to the steady state change in firing rate ( $\mathrm{Y}$ in Fig. 12A) (Lisberger and Pavelko, 1986). Figure $12 B$ plots the value of the DI for each afferent as a function of its normalized threshold current. There was considerable variation in this relationship, but the afferents with the lowest thresholds for electrical stimuli tended to have high values of DI, reflecting phasic responses during the rapid head turns. In contrast, the afferents with the highest thresholds for electrical stimulation had values of DI close to 1 , representing 


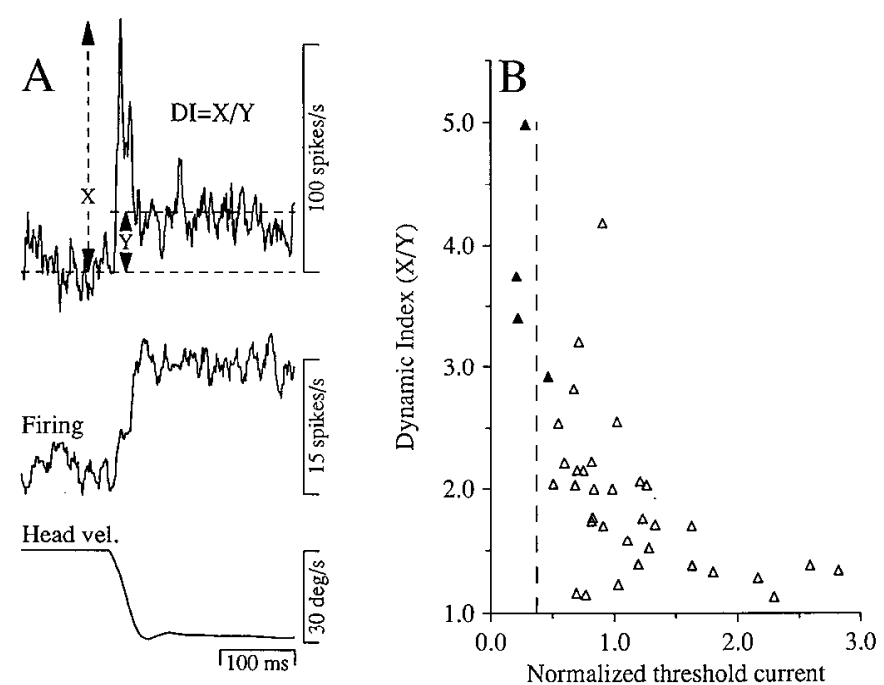

Figure 12. The relationship between normalized threshold current and the time course of afferent responses to rapid changes in head velocity. $A$, The bottom trace shows the trajectory of head velocity for a rapid change in head velocity toward the side of recording. The middle trace shows the average firing rate during the same stimulus for an afferent with regular spontaneous discharge and a normalized threshold current of 2.3. The top trace shows the average firing rate during rapid changes in head velocity for an afferent with irregular spontaneous discharge and a normalized threshold current of 0.25 . DI was calculated as $X / Y$, where $X$ is the difference between peak firing and resting firing rate, and $Y$ is the difference between steady state firing and resting firing rate. $B$, DI is plotted as a function of normalized threshold current for all fibers that were studied during rapid changes in head velocity. The dashed vertical line shows a normalized threshold current of 0.37 . Higher values of DI indicate afferent responses that were more phasic. The solid symbols show data for afferents with irregular spontaneous discharge and the open symbols show data for afferents with regular or intermediate spontaneous discharge.

tonic responses to rapid head turns. Afferents with low scnsitivities to head velocity but large phase leads during sinusoidal stimulation at $0.5 \mathrm{~Hz}$ (solid symbols) were a subset of those that had large phasic responses. Power law regression gave a best fit for the relation between DI for natural stimulation and normalized threshold for electrical activation of primary afferents: $\mathrm{DI}=0.95 T^{-0.39}(r=0.74)$.

\section{The latency for electrical activation of afferents}

Primary vestibular afferents responded to electrical stimulation with latencies that varied from 0.6 to $1.5 \mathrm{msec}$. In monkey $\mathrm{T}$ (Fig. 13A) there was no clear difference between the latencies of afferents with irregular spontaneous discharge (solid symbols) and those with intermediate or regular spontaneous discharge (open symbols). In monkey $\mathrm{U}$ (Fig. 13B), the longest latencies were recorded in afferents with intermediate or regular spontaneous discharge (open symbols) and all those with irregular spontaneous discharge (solid symbols) had latencies of $1.1 \mathrm{msec}$ or less. Logarithmic regression failed to reveal a statistically significant relationship between latency and normalized threshold current in either monkey ( $p>0.2)$. We suspect that the exact location of the stimulating electrodes in the superior canal was responsible for the small difference between the two monkeys in the latencies of afferent responses to electrical stimuli.

We have estimated the conduction velocities of our sample of afferents by measuring the distance between the stimulating and recording electrodes in a dissection of the middle fossa of one monkey after it had been killed. The distance from the

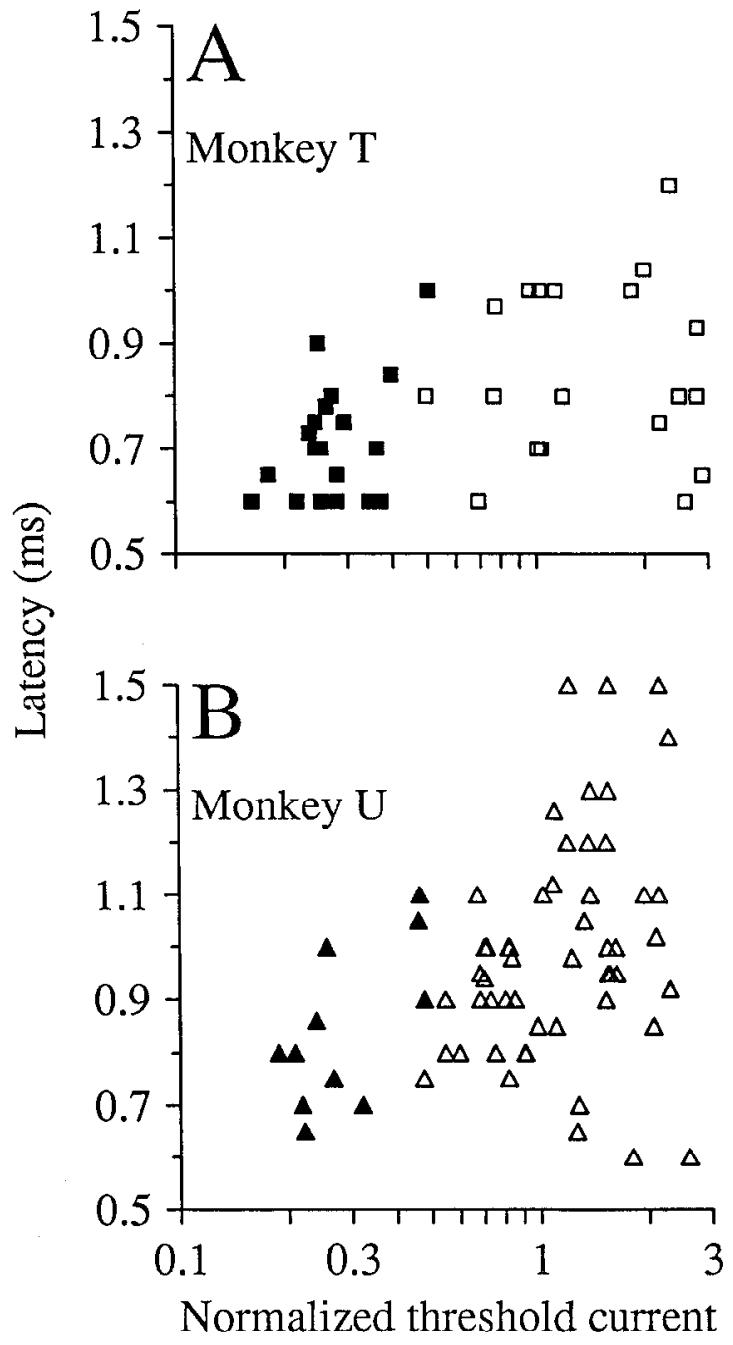

Figure 13. The latency of afferent responses to stimulation with a single stimulus pulse at threshold. Each graph plots latency as a function of the normalized threshold current. Data are plotted separately for monkey $\mathrm{T}(A)$ and monkey $\mathrm{U}(B)$. Solid symbols show the latencies for afferents with irregular spontaneous discharge. Open symbols show the latencies for afferents with intermediate and regular spontaneous discharge.

ampulla of the horizontal semicircular canal to the recording electrode was approximately $12 \mathrm{~mm}$. Thus, our measurements of latency agree well with those of Goldberg and Fernandez (1977). In horizontal canal afferents from the squirrel monkey, they recorded latencies that ranged from 0.2 to $0.5 \mathrm{msec}$ for a conduction distance of 4.1-4.8 $\mathrm{mm}$ from the site of stimulation to Scarpa's ganglion. For our experiments, both the conduction distances and the maximum and minimum latencies were about three times larger. If we assumed that the entire latency results from axonal conduction, then the latencies we recorded implied that the conduction velocity of the afferents ranged from 8 to $20 \mathrm{~m} / \mathrm{sec}$. However, these conduction velocities must be considered as lower limits because some of the latency may reside in the time required to bring the afferents to threshold. We expect that the latencies would have been shorter if we had been able to measure them at higher currents. We were not able to do so because we could not separate the axonal spike from the evoked field potential when the current was high enough to evoke a spike for every stimulus pulse. 


\section{Recovery functions of afferents}

The current required to activate an afferent is related to its $\mathrm{CV}^{*}$ and to the time of the stimulus pulse in relationship to the previous spike (Goldberg et al., 1984). For 20 afferents with $\mathrm{CV}^{*}$ ranging from 0.02 to 0.48 , we estimated the threshold current for a range of delays between the preceding action potential and the stimulus pulse. Figure 14 plots the normalized threshold current as a function of the delay between the preceding action potential and the stimulus, expressed as a percentage of the mean ISI. Each curve shows data from one afferent. For each afferent, the normalized threshold current decreased slightly but consistently as the interval between the previous spike and the stimulus was increased. However, this decrease did not cause a large change in threshold or a significant change in the recruitment order of afferents if the stimulus occurred at delays between $30 \%$ and $55 \%$ of the ISI. For delays longer than $60 \%$ of the ISI, the threshold began to decrease more sharply in the fibers that were studied at these delays. We did not perform this experiment for larger delays, because it became impossible to distinguish the evoked spikes from those that occurred naturally because the previous ISI had expired.

\section{Summary of the relationship between physiological response properties and normalized threshold current}

Table 2 divides the afferents we recorded into four groups according to their normalized threshold currents and summarizes the physiological response properties of each group. We selected the currents uscd to delimit the groups to facilitate the discussion in the next section of the contribution of different groups of afferents to the VOR. One-way ANOVA demonstrated that between-group differences were significant $(p<0.001)$ for all four parameters listed in Table 1. Pairwise $t$ tests showed that the between-group differences were statistically significant for almost every pairing of groups. Of 24 comparisons, four were not statistically significant. The sensitivity to head velocity was not significantly different at either $0.5 \mathrm{~Hz}$ or $4.0 \mathrm{~Hz}$ for comparison of afferents with normalized thresholds below 0.37 (group 1) and those with thresholds between 0.37 and 1.0 (group 2). There was no difference in either $\mathrm{CV}^{*}$ or DI for comparison of afferents with normalized threshold currents between 1.0 and 2.2 (group 3 ) and those with thresholds above 2.2 (group 4).

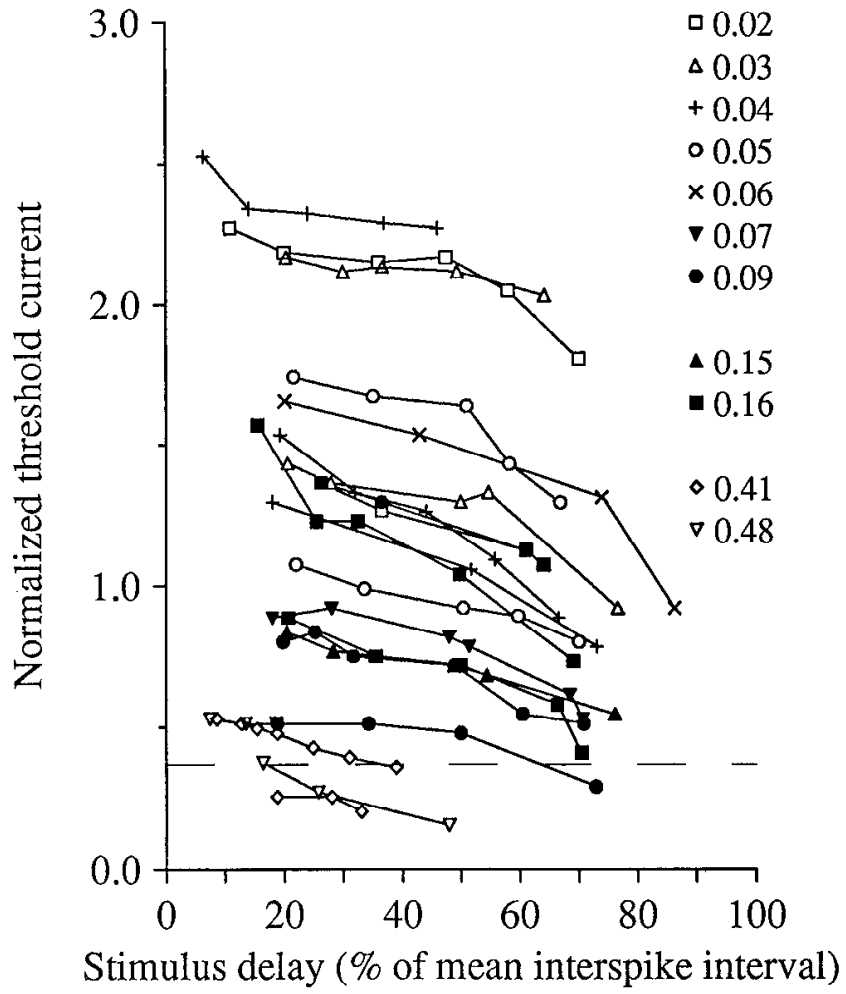

Figure 14. The recovery functions for a sample of afferents. Each set of connected points shows the data for one afferent and plots the normalized threshold current as a function of the delay between the previous spike and the application of the electrical stimulus. The delay is expressed as a percentage of the mean ISI. The key on the right of the graph identifies afferents by their normalized coefficient of variation $\left(\mathrm{CV}^{*}\right)$. The horizontal dashed line shows a normalized threshold current of 0.37 .

\section{Discussion}

Our experiments have used electrical stimulation of the vestibular apparatus to reveal a correlation among stimulation current, the physiological response properties of horizontal semicircular canal afferents that are activated by that current, and the horizontal eye velocity evoked by the stimulus. As the current was increased, there was an orderly relationship between

Table 2. Summary of the correlation among the physiological properties of afferents, their normalized threshold currents, and their contribution to the VOR

\begin{tabular}{lllll} 
& Group 1 & Group 2 & Group 3 & Group 4 \\
\hline Normalized threshold current & $0-0.37$ & $0.37-1.0$ & $1.0-2.2$ & $>2.2$ \\
CV* & $0.45 \pm 0.06$ & $0.145 \pm 0.14$ & $0.047 \pm 0.02$ & $0.033 \pm 0.008$ \\
& $(n-25)$ & $(n-34)$ & $(n-37)$ & $(n=10)$ \\
Sensitivity at $0.5 \mathrm{~Hz}$ & $0.71 \pm 0.36$ & $0.80 \pm 0.39$ & $0.42 \pm 0.28$ & $0.20 \pm 0.07$ \\
& $(n-25)$ & $(n-33)$ & $(n=36)$ & $(n=10)$ \\
Sensitivity at $4.0 \mathrm{~Hz}$ & $1.61 \pm 0.49$ & $1.39 \pm 0.83$ & $0.55 \pm 0.42$ & $0.18 \pm 0.06$ \\
& $(n=14)$ & $(n=20)$ & $(n=24)$ & $(n=8)$ \\
DI & $4.04 \pm 0.83$ & $2.24 \pm 0.74$ & $1.66 \pm 0.38$ & $1.28 \pm 0.13$ \\
& $(n=3)$ & $(n=17)$ & $(n=13)$ & $(n=3)$ \\
Contribution to VOR & $0 \%$ & $50 \%$ & $48 \%$ & $2 \%$
\end{tabular}


the stimulation current and (1) the size of the evoked eye movements, (2) the effect of changes in the gain of the VOR on the evoked eye movements, and (3) the recruitment of afferents with different physiological properties. In our Discussion, we will use these parallel effects to deduce the contributions of different vestibular afferents to the VOR before and after motor learning. It is the correlations between these parallel effects that provide the novel results and conclusions from our article. Although previous studies used different species and somewhat different physiological measures, they laid the foundations for our findings on the correlations between thresholds for electrical stimulation and physiological responses in vestibular primary afferents (Goldberg et al., 1984, 1987; Baird et al., 1988).

\section{Use of electrical stimulation to study the VOR}

Our conclusions are based on responses of the VOR pathways to single electrical pulses and brief trains of pulses applied to the superior semicircular canal. Electrical stimulation has several advantages over natural stimulation for determining which afferents contribute to the VOR. First, we have shown here that variation of the stimulation current affords systematic control over which afferents from the horizontal canal are activated. In contrast, there is no evidence of a threshold for natural vestibular stimulation; any head turn in yaw modulates the firing rate of all afferents from the horizontal semicircular canals. Second, electrical stimulation activates all afferents at latencies that range from 0.6 to $1.5 \mathrm{msec}$, creating a nearly synchronous volley in the vestibular afferents from the horizontal canal. For natural head turns, in contrast, the primary afferents respond with latencies that range widely from 4 to $18 \mathrm{msec}$ (Lisberger and Pavelko, 1986). Third, electrical stimulation bypasses the dynamics of the mechanical vestibular transduction apparatus and activates primary afferent fibers directly (Goldberg et al., 1984), so the relationship between stimulus parameters and the evoked eye movement provides direct information about how vestibular inputs are processed in the central vestibular pathways.

We think we have avoided the potential problems and disadvantages of using electrical stimulation of the labyrinth. We have demonstrated here that implants in the superior semicircular canal provide low-threshold activation of afferents from the horizontal canal without compromising the responses of horizontal canal afferents to head turns. Although electrical stimulation would have been even more effective if we had placed electrodes in the horizontal canal, our experience with such electrodes demonstrated that this procedure plugged the horizontal canal (Broussard et al., 1992). This earlier study also showed that the horizontal component of the evoked eye movements has the same properties whether the stimulating electrode is implanted in the horizontal canal or in the superior canal.

\section{Physiological properties of afferents that contribute to the VOR}

Our data imply that a group of afferents with low electrical thresholds and low sensitivities to head velocity make little or no contribution to the VOR. As a shorthand to describe these afferents, we refer to them as those with normalized threshold currents below 0.37 , even though one afferent in our sample could not be categorized so neatly. Taken at face value, our data show that afferents with normalized thresholds below 0.37 contribute at most $10 \%$ of the vestibular inputs to the VOR evoked by electrical stimulation of the labyrinth. For compatibility with our methods for determining the threshold current for activation of afferents, however, we prefer to base our conclusions about the threshold current for evoking any eye movement on experiments in which the stimulus pulse was synchronized to the previous spike in all the afferents. This was achieved by applying a pair of closely spaced pulses to the vestibular apparatus and evaluating the eye movement evoked by a second pulse. We found that no eye movement was evoked by the second pulse when the first pulse activated all the afferents and the second pulse provided a normalized stimulation current of 0.26 or below. In our experiments with single pulses, we think that the lack of synchrony between the stimulus and the ongoing activity of afferents allowed small eye movements to be evoked at the lowest stimulation currents. When applied at a rate of $5 \mathrm{~Hz}$, single pulses could occur at any time in relation to the spontaneous spikes of each individual afferent. For each individual pulse, some afferents would be about to emit a spontaneous spike at the time of the stimulus and would therefore have thresholds lower than we measured in our recordings from vestibular afferents. It follows that a few afferents would have been activated by single pulses at normalized stimulation currents below 0.37 , even though their thresholds were above 0.37 when the stimulus was triggered off a previous spike. Thus, the presence of small eye movements in response to single electrical pulses at normalized currents below 0.37 could be an artifact arising from the use of asynchronous single pulse stimuli. We do not think it is likely that the absence of evoked eye movements at normalized stimulation currents below 0.37 could be explained by a failure to bring central neurons above firing threshold. This explanation would be plausible if interneurons in the VOR pathways did not fire spontaneously. However, the best candidates for interneurons in the disynaptic VOR pathways, position-vestibular-pause cells (Scudder and Fuchs, 1992) and flocculus target neurons (Lisberger and Pavelko, 1988), are spontaneously active at rates of 50-150 spikes/sec for fixation within the central $20^{\circ}$. Therefore, even a small input at low stimulation currents should influence the probability of firing for the VOR interneurons and should evoke a small eye movement.

We think that the afferents with the lowest normalized threshold currents (less than 0.37 ) do not contribute to the VOR at all. For these afferents, the relationship between the sensitivity to head velocity for sinusoidal stimulation at $0.5 \mathrm{~Hz}$ and normalized threshold current did not fit with the rest of our sample of afferents. As summarized in Table 2, the afferents with thresholds below 0.37 consist only of irregularly discharging fibers with a mean $\mathrm{CV}^{*}$ of 0.45 . Although we recorded from only a few such afferents during rapid changes in head velocity in the present study, the data of Lisberger and Pavelko (1986) agree with our finding that the afferents with the most irregular spontaneous discharge exhibit (1) large phase leads with respect to head velocity and (2) phasic-tonic responses to rapid changes in head velocity (mean DI $=4.04$ in our sample). Morphophysiological studies in the chinchilla (Baird et al., 1988; Fernandez et al., 1988) and the monkey (Lysakowski et al., 1988) have shown that the irregular afferents with low sensitivities to head velocity form a separate morphological group that innervates hair cells in the most central regions of the crista with calyceal endings. Thus, comparison of their data with ours implies that the VOR pathways do not receive inputs from afferents that make only calyceal endings on hair cells. This conclusion is entirely consistent with the fact that the VOR is not altered by reversible ablation of the primary afferents with irregular spontaneous discharge (Minor and Goldberg, 1991). 
Our data on the eye movements evoked by single electrical pulses show that afferents with normalized threshold currents between 0.37 and 1.0 are responsible for about half of the rise in the peak eye velocity to its maximum value. These afferents exhibited response properties that spanned the entire range of our sample. As summarized in Table 2, they can have spontaneous discharges that are irregular, intermediate, or regular; they have a high mean and a wide range of sensitivities to head velocity for sinusoidal head motion at 0.5 and $4 \mathrm{~Hz}$; and their responses to rapid head turns range from tonic to highly phasic. Afferents with normalized threshold currents between 1.0 and 2.2 provided the other half of the increase in peak eye velocity toward its maximum value. The afferents recruited over this range of currents had a narrower range of physiological properties, including regular spontaneous discharge, low values of sensitivity to head velocity, and more nearly tonic responses during rapid head turns. Comparison of the physiological responses of afferents in our sample with the morphophysiological data (Baird et al., 1988; Fernandez. et al., 1988; Lysakowski et al., 1988) suggests that the afferents recruited at normalized threshold currents between 0.37 and 2.2 make dimorphic endings on hair cells, with both calyceal and bouton terminals.

The response properties of afferents with normalized threshold currents between 0.37 and 1.0 suggest a possible resolution to a disagreement about whether the VOR pathways receive any inputs from afferents with phasic-tonic responses during rapid head turns. In an earlier study from our laboratory, Lisberger and Pavelko (1986) suggested that afferents with phasic-tonic responses played an important role in the VOR. Minor and Goldberg (1991) equated phasic-tonic responses with irregular spontaneous discharge and, because they had demonstrated that irregular afferents made no contribution to the VOR, developed a model that could account for the data of Lisberger (1984) and Lisberger and Pavelko (1986) without assuming that afferents with phasic-tonic responses contribute to the VOR. Our data show that afferents with normalized threshold currents between 0.37 and 1.0 make a substantial contribution to the VOR and all but two of the afferents we recorded in this group had a strong phasic component in the firing rate evoked by rapid head turns. Thus, afferents with intermediate or even with regular discharge can have phasic-tonic responses during rapid head turns and could contribute significantly to the VOR without contradicting the conclusion of Minor and Goldberg (1991) that the VOR does not derive inputs from vestibular afferents with irregular spontaneous discharge.

Our experiments provided no evidence that afferents with normalized threshold currents above 2.2 make a substantial contribution of the VOR. These afferents were the most homogencous group we encountered. They had very regular spontaneous firing rates with a mean $\mathrm{CV}^{*}$ of 0.033 , they exhibited very low sensitivities to sinusoidal vestibular stimulation at low or high frequencies, and they showed tonic responses to rapid changes in head velocity (Table 2). Comparison with morphophysiological data (Baird et al., 1988) suggests that the afferents with normalized threshold currents greater than 2.2 may be an anatomically distinct group corresponding to the bouton fibers that innervate the periphery of the crista.

Electrical and natural stimulation of the vestibular system activate primary afferents and central vestibular pathways in completely different ways. Uncertainty about exactly how central neurons respond to electrical stimuli at different currents raises a number of alternative interpretations of our data. First, afferents with normalized threshold currents above 2.2 could still be important in the VOR evoked by natural head turns, even though they do not make a clear contribution to the eye movements evoked by single pulses or trains of electrical stimuli. A single electrical pulse at a normalized current of 2.2 may cause saturation at some stage along the VOR pathways, for example, by causing an action potential in every motoneuron or in every secondary vestibular neuron. If this were the case, then further increases in current would have no further effect on the evoked eye velocity, even if the afferents with higher thresholds projected into VOR pathways. Trains of electrical stimuli probably approximate better the input provided by natural head turns. However, the synchrony of the afferent responses to each pulse in the train leaves open the possibility that there is saturation at low currents in the early stages of pathways that receive inputs from afferents with high-threshold currents. Thus, our approach may underestimate the contribution to the VOR of afferents with regular spontaneous discharge and high electrical thresholds. Second, activation of the vestibular apparatus with a single electrical pulse may activate preferentially a subset of VOR pathways, for example, those with the smallest number of intervening synapses. The distribution of afferent inputs revealed by our data may emphasize the contribution of the disynaptic VOR pathways, while pathways with more than two synapses may receive a different distribution of afferent inputs. It is difficult to use our data to draw final conclusions about the afferents that contribute to the VOR, because the multisynaptic pathways probably make a proportionately larger contribution to the VOR evoked by natural stimulation than to the eye movements evoked by a single electrical pulse. This problem may be partially mitigated by our results with trains of stimuli. Trains should activate multisynaptic pathways more effectively than do single electrical pulses, but the evoked eye velocity increased over the same range of currents for both single pulses and trains of pulses. Finally, it is not clear how increases in stimulation current affect the responses of individual neurons. In decerebrate cats, stimulation at high currents evokes double discharges in second-order neurons (Precht and Shimazu, 1965), and the second discharge could contribute to the growth of the evoked eye velocity as current is increased. It may, however, still be true that increases in stimulation current elicit a second discharge in central neurons as a direct consequence of activating afferents with more regular spontaneous discharge. The issues raised in this paragraph can be resolved by studying the responses of central neurons and the eye movements evoked by electrical stimulation at the same time.

Two previous studies have used the electrical thresholds of different afferents to provide information about the propertics of afferents that project into VOR pathways. Goldberg et al. (1987) showed that $75 \%$ of secondary vestibular neurons in the vestibular nucleus receive monosynaptic inputs from afferents with a broad range of response properties. Highstein et al. (1987) subdivided the population of secondary neurons according to their projection sites and found that $57 \%$ of identified interneurons in the VOR pathways received monosynaptic inputs from afferents with a broad range of response properties. However, Highstein et al. (1987) interpreted the wide range of vestibular inputs to VOR interneurons as contamination of their sample with other "pause-burst" neurons and concluded that VOR pathways receive input mainly from afferents with regular spontaneous discharge. Our data disagree with the interpretation 
of Highstein et al. (1987), but agree with their data as well as with those of Goldberg et al. (1987). At least for the VOR pathways that are activated by a single electrical pulse, the inputs for the VOR appear to arise abundantly from afferents that show intermediate electrical thresholds as well as a wide range of physiological response properties and discharge regularities.

\section{Roles of different vestibular afferents for motor learning in the VOR}

Previous studies have identified one component of the VOR that is modified in association with changes in the gain of the VOR and one that is not modified. When the stimulus is a rapid head turn, the two components differ in their latency (Lisberger, 1984) and in the dynamics of the signals they transmit to the extraocular motoneurons (Lisberger and Pavelko, 1986). When the stimulus is a single electrical pulse, the two components differ on the dynamics of the signals they transmit, but not in their latency (Broussard et al., 1992; present results). The data in the present report suggest that the modified and unmodified components of the VOR are driven by different (but likely overlapping) subsets of the vestibular primary afferents. For example, the existence of a significant evoked eye velocity but the relatively small effect of motor learning on the eye movements evoked at normalized currents below 0.72 demonstrates that afferents with normalized threshold currents between 0.37 and 0.72 make a large contribution to the unmodified component of the VOR and a smaller contribution to the modified component. Our data on the eye movements evoked by trains of electrical pulses show that activation of different populations of afferents generates eye movements with quite different time courses, suggesting that the different afferents project into pathways that have quite different dynamics. Finally, our data suggest that different groups of afferents make contributions to the modified VOR at different latencies after the onset of the stimulus. Affcrents with normalized threshold currents below 1.2 appear to make their largest contribution to the eye movements up to $22 \mathrm{msec}$ after the application of an electrical stimulus while afferents with higher thresholds contribute at later times. We conclude that afferents with higher thresholds for electrical stimulation project into pathways that have a prolonged effect on motor output and/or contribute to the VOR at longer latencies.

Our data demonstrate that motor learning in the VOR is mediated by afferents with a wide range of physiological response properties. Although we cannot provide final answers on the exact identity of afferents that contribute to the modified and unmodified components of the VOR, we want to emphasize that it will be critical to view vestibular afferents as a continuum. For example, the wide distributions of the physiological responses of afferents with normalized thresholds between 0.37 and 2.2 gave no clear evidence that they should be broken into subgroups. We have defined specific groups here only to assist in the interpretation of our data, not because we think the different groups have entirely distinct functions. It will be necessary to evaluate the relative contributions of afferents across the spectrum of physiological response properties, rather than attempting to define function according to qualitative and artificial divisions into discrete groups.

\section{Implications for the mechanisms of motor learning}

The eye movements evoked by natural stimuli or by trains of electrical stimuli are affected much more profoundly by changes in the gain of the VOR than are the eye movements evoked by single electrical pulses (Broussard et al., 1992; present results). We think this reflects a basic difference in the mode of activation of the VOR pathways by different stimulation procedures and that it may tell us something about the mechanisms of motor learning in the VOR. If changes in the gain of the VOR were mediated simply by changes in synaptic weight, like those thought to occur in long-term potentiation (Bliss and Collingridge, 1993), then motor learning ought to induce large changes in the eye movements evoked by a single electrical pulse. The existence of small changes starting at the earliest part of these responses (Broussard et al., 1992) is consistent with the idca that part of the mechanism of motor learning in the VOR involves changes in synaptic weight in the disynaptic VOR pathways in the brainstem. However, neurons possess multiple mechanisms that operate on a time scale of tens of milliseconds to provide temporal integration of incoming synaptic currents. Integration mechanisms could reside either in the machinery of synaptic transmission or in the conversion of synaptic currents into spike trains. Single pulses, which cause a single extra spike in interneurons in the VOR pathways (Broussard and Lisberger, 1992; Scudder and Fuchs, 1992), may bypass most of these mechanisms. In contrast, trains of stimulus pulses would have access to mechanisms that are responsible for temporal integration.

Comparison of the eye movements evoked by single pulses and trains of pulses raises the possibility that the processing of vestibular inputs for the VOR depends partly on cellular mechanisms that perform temporal integration of synaptic in puts that occur closely in time. For example, the profound effect of stimulation current on the trajectory of the eye velocity evoked by trains of pulses suggests that afferents with higher thresholds for electrical stimuli are processed by mechanisms that emphasize temporal integration. The effect of motor learning on the trajectory of the eye velocity evoked by trains of electrical pulses at some stimulation currents suggests that motor learning in the VOR is implemented partly by modification of the cellular mechanisms that perform these temporal integrations.

\section{The role of vestibular afferents in different vestibulomotor reflexes}

Our findings provide support for the thesis (Bilotto et al., 1982) that vestibular afferents are channeled such that they provide inputs to reflex pathways for which their signals are well matched. For example, afferents with normalized threshold currents below 0.37 do not appear to contribute to the VOR but they have dynamic response properties that are more suitable to allow the VCR to overcome the inertial load presented by the head. Bilotto et al. (1982) found that the phase shift between head velocity and EMG of neck muscles is in good agreement with the phase shift of the signals carried by these afferents, while the phase shift between head velocity and the EMG of the lateral rectus muscle was much smaller. Our data suggest that afferents with normalized threshold currents between 0.37 and 2.2 project into VOR pathways, but may be channeled so that those with lower thresholds make stronger projections into unmodified pathways while those with higher thresholds make stronger projections into modified VOR pathways. Finally, afferents with normalized threshold currents above 2.2 may not provide strong inputs to the VOR pathways, but their tonic firing in phase with head velocity may be well suited to provide some of vestibular signals that descend in the lateral vestibulospinal tract from Deiters nucleus to limb muscles. For example, Hirai et al. (1979) dem- 
onstrated that the VSR exhibits a low vestibular sensitivity at all frequencies and shows substantial phase lag at high frequencies rather than the phase lead exhibited by the VCR.

Almost every sensory system is subserved by afferents with a broad range of functional properties. For example, the primary muscle spindle afferents of the decerebrate cat have an irregular spontaneous discharge pattern and exhibit increased sensitivity and phase lead for sinusoidal modulation of muscle length at high frequencies (Matthews and Stein, 1969a). The secondary endings have a more regular discharge pattern (Matthews and Stein, 1969b) and show lower sensitivities and less phase lead during sinusoidal modulation of muscle length (Matthews and Stein, 1969a). The two types of muscle stretch afferents subserve complementary functions that allow spinal reflexes to exert fine control over muscle length and tension under a wide range of conditions. In the vestibular system, different but overlapping groups of vestibular afferents subserve complementary functions in different vestibulomotor reflexes. An understanding of the spccific functions of different afferents in the vestibular system should provide some guidelines for determining, in other sensory systems, how inputs with different physiological response properties are distributed to multiple effector pathways.

\section{References}

Baird RA, Desmadryl G, Fernandez C, Goldberg JM (1988) The vestibular nerve of the chinchilla. II. Relation between afferent response properties and peripheral innervation patterns in the semicircular canals. J Neurophysiol 60:182-203.

Bilotto G, Goldberg J, Peterson BW, Wilson VJ (1982) Dynamic properties of vestibular reflexes in the decerebrate cat. Exp Brain Res 47:343-352.

Blanks RHI, Estes MS, Markham CH (1975) Physiologic characteristics of vestibular first-order canal neurons in the cat. II. Response to constant angular acceleration. J Neurophysiol 38:1250-1268.

Bliss TVP, Collingridge GL (1993) A synaptic model of memory: longterm potentiation in the hippocampus. Nature 361:31-39.

Broussard DM, Lisberger SG (1992) Vestibular inputs to brain stem neurons that participate in motor learning in the primate vestibuloocular reflex. J Neurophysiol 68:1906-1909.

Broussard DM, Brontë-Stewart HM, Lisberger SG (1992) Expression of motor learning in the response of the primate vestibuloocular reflex pathway to electrical stimulation. J Neurophysiol 67:1493-1508.

Estes MS, Blanks RHI, Markham CH (1975) Physiologic characteristics of vestibular first-order canal neurons in the cat. I. Response plane determination and resting discharge characteristics. J Neurophysiol 38:1232-1249.

Ezure K, Sasaki S (1978) Frequency response analysis of vestibularinduced neck reflex in cat. I. Characteristics of neural transmission from horizontal semicircular canal to neck motoneurons. J Neurophysiol 41:445-458.

Fernandez C, Goldberg JM (1971) Physiology of peripheral neurons innervating semicircular canals of the squirrel monkey. II. Response to sinusoidal stimulation and dynamics of peripheral vestibular system. J Neurophysiol 34:661-675.

Fernandez C, Baird RA, Goldberg JM (1988) The vestibular nerve of the chinchilla. I. Peripheral innervation patterns in the horizontal and superior semicircular canals. J Neurophysiol 60:167-181.

Goldberg JM, Fernandez C (1971a) Physiology of peripheral neurons innervating semicircular canals of the squirrel monkey. I. Resting discharge and response to constant angular accelerations. J Neurophysiol 34:635-660.

Goldberg JM, Fernandez C (1971b) Physiology of peripheral neurons innervating semicircular canals in the squirrel monkey. III. Variation among units in their discharge properties. J Neurophysiol 34:676684.

Goldberg JM, Fernandez C (1977) Conduction times and background discharge of vestibular afferents. Brain Res 122:545-550.

Goldberg JM, Smith CE, Fernandez C (1984) Relation between discharge regularity and responses to externally applied galvanic currents in vestibular nerve afferents of the squirrel monkey. J Neurophysiol 51:1236-1256.

Goldberg JM, Highstein SM, Moschovakis AK, Fernandez C (1987) Inputs from regularly and irregularly discharging vestibular nerve afferents to secondary neurons in the vestibular nuclei of the squirrel monkey. I. An electrophysiological analysis. I Neurophysiol 58:700718.

Highstein SM, Goldberg JM, Moschovakis AK, Fernandez C (1987) Inputs from regularly and irregularly discharging vestibular nerve afferents to secondary neurons in the vestibular nuclei of the squirrel monkey. II. Correlation with output pathways of secondary neurons. J Neurophysiol 58:719-738.

Hirai N, Hwang JC, Wilson VJ (1979) Comparison of dynamic properties of canal-evoked vestibulospinal reflexes of the neck and forelimb in the decerebrate cat. Exp Brain Res 36:393-397.

Judge SJ, Richmond BJ, Chu FC (1980) Implantation of magnetic search coils for measurement of eye position: an improved method. Vision Res 20:535-538.

Lisberger SG (1984) The latency of pathways containing the site of motor learning in the monkey vestibulo-ocular reflex. Science 225 : 74-76.

Lisberger SG, Miles FA (1980) Role of primate medial vestibular nucleus in long-term adaptive plasticity of vestibuloocular reflex. $J$ Neurophysiol 43:1725-1745.

Lisberger SG, Pavelko TA (1986) Vestibular signals carried by pathways subserving plasticity of the vestibulo-ocular reflex in monkeys. J Neurosci 6:346-354.

Lisberger SG, Pavelko TA (1988) Brain stem neurons in modified pathways for motor learning in the primate vestibulo-ocular reflex. Science 242:771-773.

Lisberger SG, Sejnowski TJ (1992) Motor learning in a recurrent network model based on the vestibulo-ocular reflex. Nature 360:159161 .

I.ysakowski A, Minor LB, Fernandez C, Goldberg JM (1988) Physiological identification of calyx, dimorphic and bouton afferents in the vestibular nerve of the squirrel monkey. Soc Neurosci Abstr 14:172.

Matthews PBC, Stein RB (1969a) The sensitivity of muscle spindle afferents to small sinusoidal changes of length. J Physiol (Lond) 200: $723-743$.

Matthews PBC, Stein RB (1969b) The regularity of primlary and secondary muscle spindle afferent discharges. J Physiol (Lond) 202:5983.

Melvill Jones G, Milsum JH (1970) Characteristics of neural transmission from the semicircular canal to the vestibular nuclei of cats. J Physiol (Lond) 209:295-316.

Merigan WH, Maunsell JHR (1993) How parallel are the primate visual pathways? Annu Rev Neurosci 16:369-402.

Miles FA, Braitman DJ (1980) Long-term adaptive changes in primate vestibulo-ocular reflex. II. Electrophysiological observations on semicircular canal primary afferents. J Neurophysiol 43:1426-1436.

Miles FA, Eighmy BB (1980) Long-term adaptive changes in primate vestibulo-ocular reflex. I. Behavioral observations. J Neurophysiol 43:1406-1425.

Minor LB, Goldberg JM (1991) Vestibular-nerve inputs to the vestibulo-ocular reflex: a functional-ablation study in the squirrel monkey. J Neurosci 11:1636-1648.

Peterson BW, Bilotto G, Goldberg J, Wilson VJ (1981) Dynamics of vestibulo-ocular, vestibulocollic and cervicocollic reflexes. Ann NY Acad Sci 374:395-402.

Precht W, Shimazu H (1965) Functional connections of tonic and kinetic vestibular neurons with primary vestibular afferents. J Neurophysiol 28:1014-1028.

Robinson DA (1970) Oculomotor unit behavior in the monkey. J Neurophysiol 33:393-404.

Scudder CA, Fuchs AF (1992) Physiological and behavioral identification of vestibular nucleus neurons mediating the horizontal vestibuloocular reflex in trained rhesus monkeys. J Neurophysiol 68:244264.

Shimazu H, Precht W (1965) Tonic and kinetic responses of cat's vestibular neurons to horizontal angular acceleration. J Neurophysiol 28:991-1013.

Shinoda Y, Yoshida K (1974) Dynamic characteristics of responses to horizontal head angular acceleration in vestibuloocular pathway in the cat. J Neurophysiol 37:653-673. 
Skavenski AA, Robinson DA (1973) Role of abducens neurons in vestibuloocular reflex. J Neurophysiol 36:724-738.

Tomko DL, Peterka RJ, Schor RH, O'Leary DP (1981) Response dynamics of horizontal canal afferents in barbiturate-anesthetized cats. J Neurophysiol 45:376-396.
Wilson VJ, Peterson BW, Fukushima K, Hirai N, Uchino Y (1979) Analysis of vestibulocollic reflexes by sinusoidal polarization of vestibular afferent fibers. J Neurophysiol 42:331-346.

Wurtz RH (1969) Response of striate cortex neurons to stimuli during rapid eye movements in the monkey. J Neurophysiol 32:975-986. 